August 2005

\title{
Top and Higgs Flavor Changing Neutral Couplings in two Higgs Doublets Model
}

\author{
Abdesslam Arhrib \\ Physics Division, National Center for Theoretical Sciences, \\ PO-Box 2-131 Hsinchu, Taiwan 300 and \\ Département de Mathématiques, Faculté des Sciences et Techniques \\ B.P 416 Tanger, Morocco.
}

\begin{abstract}
We study various channels of top and Higgs Flavor Changing Neutral Couplings (FCNC) in the two Higgs doublet Model with natural flavor conservation (2HDM-I and 2HDM-II). We update known results about $t \rightarrow c \gamma, c Z, c g$ and comment on $t \rightarrow$ $c h^{0}$. The decays $t \rightarrow c h^{0}$ as well as $\left\{h^{0}, H^{0}\right\} \rightarrow \bar{t} c$ are sensitive both to the bottom Yukawa coupling as well as to the trilinear scalar couplings $h^{0} H^{+} H^{-}$and $H^{0} H^{+} H^{-}$. After imposing unitarity constraints as well as vacuum stability conditions on scalar sector parameters, in $2 \mathrm{HDM}-\mathrm{II}$ we found that for large $\tan \beta \gtrsim 40$ and rather light charged Higgs mass $M_{H \pm} \lesssim 150 \mathrm{GeV}$, the maximum values allowed for $B r\left(t \rightarrow c h^{0}\right)$, $\operatorname{Br}\left(H^{0} \rightarrow \bar{t} c\right)$ and $\operatorname{Br}\left(h^{0} \rightarrow \bar{t} c\right)$ are: $8 \times 10^{-5}, 10^{-3}$ and $10^{-4}$ respectively. For charged Higgs mass in the range $[200,300] \mathrm{GeV}$, which can accommodate $B \rightarrow X_{s} \gamma$ constraint if one takes into account large theoretical uncertainties, the branching ratio of both $H^{0} \rightarrow \bar{t} c$ and $h^{0} \rightarrow \bar{t} c$ can still be slightly larger than $10^{-5}$. For $A^{0} \rightarrow \bar{t} c$, its branching ratio is smaller than $\approx 10^{-7}$ in both 2 HDM-I and 2 HDM-II. We study also the top-charm associated production at $e^{+} e^{-}$colliders and its $\gamma \gamma$ option as well as at muon colliders. It is found that the cross section of $\gamma \gamma \rightarrow \bar{t} c$ can be of the order $0.01 \rightarrow 0.1 \mathrm{fb}$ near threshold region, while the cross section of $e^{+} e^{-} \rightarrow \bar{t} c$ is well below $10^{-2} \mathrm{fb}$. The situation is slightly better for muon colliders where a few $\mathrm{fb}$ cross section can be reached for large $\tan \beta$ and low center of mass energy $\sqrt{s} \lesssim 500$ $\mathrm{GeV}$.
\end{abstract}




\section{Introduction}

One of the goals of the next generation of high energy colliders, such as the large hadron colliders LHC [1] or the International Linear Colliders ILC [2] or muon colliders [3], is to probe top flavor-changing neutral couplings 'top FCNC' as well as the Higgs flavorchanging neutral couplings 'Higgs FCNC'. FCNC of heavy quarks have been intensively studied both from the theoretical and experimental point of view. Such processes are being well established in Standard Model (SM) and are excellent probes for the presence of new physics effects such as Supersymmetry, extended Higgs sector and extra fermions families.

Within the SM, with one Higgs doublet, the FCNC Ztc vanish at tree-level by the GIM mechanism, and the $\gamma \bar{t} c$ and $g \bar{t} c$ couplings are zero as a consequence of the unbroken $S U(3)_{c} \times U(1)_{\text {em }}$ gauge symmetry. The Higgs FCNC $H \bar{t} c$ and $H b s$ couplings also vanish due to the existence of only one Higgs doublet. Both top FCNC and Higgs FCNC are generated at one loop level by charged current exchange, but they are very suppressed by the GIM mechanism. The calculation of the branching ratios for top decays yields the SM predictions [4], [5]:

$$
\begin{aligned}
& \mathrm{Br}(\mathrm{t} \rightarrow \mathrm{Zc})=1.3 \times 10^{-13}, \mathrm{Br}(\mathrm{t} \rightarrow \gamma \mathrm{c})=4.3 \times 10^{-13}, \mathrm{Br}(\mathrm{t} \rightarrow \mathrm{gc})=3.8 \times 10^{-11}, \\
& \operatorname{Br}(\mathrm{t} \rightarrow \mathrm{Hc})=5.6 \rightarrow 3.2 \times 10^{-14} \quad \text { for } \quad \mathrm{M}_{\mathrm{H}}=115 \rightarrow 130 \mathrm{GeV} \\
& \operatorname{Br}(\mathrm{H} \rightarrow \overline{\mathrm{t}} \mathrm{c}) \approx 1.5 \times 10^{-16} \quad\left(\operatorname{resp} 3 \times 10^{-13}\right) \quad \mathrm{M}_{\mathrm{H}}=200 \quad(\operatorname{resp} 500) \mathrm{GeV}
\end{aligned}
$$

In the last decade, there has been intensive activities to explore the top FCNC both from the experimental and theoretical point of view. Experimentally, CDF, D0 [6] and LEPII [7] collaborations have reported interesting bounds on top FCNC. These bounds are very weak but will improve in the next years, first with Tevatron Run II, and later with the future experiments such as LHC and/or ILC.

From the theoretical side, many SM extensions predict that these top and Higgs FCNC can be orders of magnitude larger than their SM values (see [8] for an overview). Among those extensions, we cite:

i) Models with exotic quarks [9], [10] where one can have branching ratios of the order $10^{-2}, 10^{-5}$ and $5 \times 10^{-4}$ respectively for $t \rightarrow c Z, t \rightarrow c \gamma$ and $t \rightarrow c g$.

ii) Two Higgs doublet models with and without Natural Flavor Conservation (2HDM-I, 2HDM-II and 2HDM-III) [4],[11],[12],[13], [14], [15], [16] where the branching ratio can be of the order $10^{-6}, 10^{-7}, 10^{-5}$ and $10^{-5}$ respectively for $t \rightarrow c Z, t \rightarrow c \gamma, t \rightarrow c g$ and $\left\{h^{0}, H^{0}\right\} \rightarrow \bar{b} s$. Note that under some assumptions on the textures of Lepton Yukawa couplings, the 2HDM can also leads to Lepton Falvor violation in Higgs boson decays see Ref. [17] for details.

iii) and a variety of supersymmetric models [18], [19], [20], [21]. In such cases, substantial enhancement can be found in R parity violating MSSM models with branching ratios of about $10^{-3}, 10^{-5}$ and $10^{-4}$ respectively for $t \rightarrow c g, t \rightarrow c \gamma$ and $t \rightarrow c Z$. The corresponding values in the MSSM with $\mathrm{R}$ parity conservation are of the order $10^{-6}$. A particular channel is the top FCNC coupling $t \rightarrow c \Phi, \Phi=h^{0}, H^{0}, A^{0}$. In the case of flavor violation induced by gluino, one can reach $10^{-4}$ branching ratio for $t \rightarrow c h^{0}$ [20]. 
Hence, top and Higgs FCNC offer a good place to search for new physics, which may manifest itself if those couplings are observed in future experiments such as LHC or ILC $[1,2]$. At LHC with low luminosity $10 \mathrm{fb}^{-1}, 8$ million $t \bar{t}$ pairs per experiment per year will be produced. This number will increase by one order of magnitude with the high luminosity option. Therefore, the properties of top quarks can be examined with significant precision at LHC. For top FCNC, it is possible to reach the following limits [1, 22]:

$$
\begin{array}{ll}
\operatorname{Br}(t \rightarrow c H) \leq 4.5 \times 10^{-5} & , \quad \operatorname{Br}(t \rightarrow c \gamma) \leq 3.7 \times 10^{-6} \\
\operatorname{Br}(t \rightarrow c Z) \leq 7.1 \times 10^{-5} & , \quad \operatorname{Br}(t \rightarrow c g) \leq 10^{-5}
\end{array}
$$

At ILC the sensitivity is less [22]::

$$
\operatorname{Br}(t \rightarrow c H) \leq 4.5 \times 10^{-5} \quad, \quad \operatorname{Br}(t \rightarrow c \gamma) \leq 7.7 \times 10^{-6}
$$

Consequently, models which can enhance these FCNC couplings close to the above limits are welcome.

The aim of this paper is to study top and Higgs FCNC couplings in the framework of the tree level Flavor Conserving two Higgs doublet Models type I and II. We update previous results for $t \rightarrow c \gamma, t \rightarrow c Z$ and $t \rightarrow c g$ [4] and revisit the decay $t \rightarrow c h^{0}$ in the light of full unitarity constraints and vacuum stability conditions on the Higgs sector. We also present our results for Higgs FCNC such as $h^{0} \rightarrow \bar{t} c, H^{0} \rightarrow \bar{t} c$ and $A^{0} \rightarrow \bar{t} c$ in $2 \mathrm{HDM}$ imposing unitarity constraints. Moreover, we address the signature of those top and Higgs FCNC couplings at $e^{+} e^{-}$Colliders, its $\gamma \gamma$ option as well as at future muon colliders. The isolated top quark signature may facilitate the search for Higgs FCNC $\Phi \rightarrow \bar{t} c$ events and also associate top-charm production.

The paper is organized as follows. In the next section, the 2HDM is introduced. Relevant couplings are given, and theoretical and experimental constraints on 2HDM parameters are discussed. In the third section, we will study the effect of $2 \mathrm{HDM}$ on top FCNC decays such as $t \rightarrow c \gamma, t \rightarrow c Z, t \rightarrow c g$ and $t \rightarrow c h^{0}$. Section four is devoted to Higgs FCNC couplings: $\operatorname{Br}(\Phi \rightarrow \bar{t} c), \Phi=h^{0}, H^{0}, A^{0}$, which are evaluated in 2HDM-I and 2HDM-II. In section 5 we investigate the top-charm associate production at $e^{+} e^{-}$, its $\gamma \gamma$ options and muon colliders. Our conclusions are given in section 6 .

\section{The $2 \mathrm{HDM}$}

Two Higgs Doublet Model $(2 \mathrm{HDM})$, is formed by adding an extra complex $S U(2)_{L} \otimes$ $U(1)_{Y}$ scalar doublet to the SM Lagrangian. Motivations for such a structure include $\mathrm{CP}$-violation in the Higgs sector [23, 24] and the fact that some models of dynamical electroweak symmetry breaking yields the 2HDM as their low-energy effective theory [25]. In particular, the Higgs sector of the Minimal Supersymmetric Standard Model (MSSM) takes the form of a constrained 2HDM. 
The most general 2HDM scalar potential which is both $S U(2)_{L} \otimes U(1)_{Y}$ and CP invariant is given by* $[26]$ :

$$
\begin{aligned}
V\left(\Phi_{1}, \Phi_{2}\right) & =\lambda_{1}\left(\left|\Phi_{1}\right|^{2}-v_{1}^{2}\right)^{2}+\lambda_{2}\left(\left|\Phi_{2}\right|^{2}-v_{2}^{2}\right)^{2}+\lambda_{3}\left(\left(\left|\Phi_{1}\right|^{2}-v_{1}^{2}\right)+\left(\left|\Phi_{2}\right|^{2}-v_{2}^{2}\right)\right)^{2}+ \\
& \lambda_{4}\left(\left|\Phi_{1}\right|^{2}\left|\Phi_{2}\right|^{2}-\left|\Phi_{1}^{+} \Phi_{2}\right|^{2}\right)+\lambda_{5}\left[\Re e\left(\Phi_{1}^{+} \Phi_{2}\right)-v_{1} v_{2}\right]^{2}+\lambda_{6}\left[\Im m\left(\Phi_{1}^{+} \Phi_{2}\right)\right]^{2}
\end{aligned}
$$

where $\Phi_{1}$ and $\Phi_{2}$ have weak hypercharge $\mathrm{Y}=1, v_{1}$ and $v_{2}$ are respectively the vacuum expectation values of $\Phi_{1}$ and $\Phi_{2}$ and the $\lambda_{i}$ are real-valued parameters. Note that this potential violates the discrete symmetry $\Phi_{i} \rightarrow-\Phi_{i}$ only softly by the dimension two term $\lambda_{5} \Re e\left(\Phi_{1}^{+} \Phi_{2}\right)$. The hard breaking terms (dimension four) of the discrete symmetry have been set to zero.

The above scalar potential has 8 independent parameters $\left(\lambda_{i}\right)_{i=1, \ldots, 6}, v_{1}$ and $v_{2}$. After electroweak symmetry breaking, the combination $v_{1}^{2}+v_{2}^{2}$ is thus fixed by the electroweak scale through $v_{1}^{2}+v_{2}^{2}=\left(2 \sqrt{2} G_{F}\right)^{-1}$. We are left then with 7 independent parameters. Meanwhile, three of the eight degrees of freedom of the two Higgs doublets correspond to the 3 Goldstone bosons $\left(G^{ \pm}, G^{0}\right)$ and the remaining five become physical Higgs bosons: $H^{0}$, $h^{0}$ (CP-even), $A^{0}$ (CP-odd) and $H^{ \pm}$. Their masses are obtained as usual by diagonalizing the mass matrix. The presence of charged Higgs bosons will give new contributions to the one-loop induced top and Higgs FCNC couplings (see Fig. $1 d_{11} \rightarrow d_{18}$ ).

It is possible to write the quartic coupling $\lambda_{i}$ in terms of physical scalar masses, mixing angles $\tan \beta, \alpha$ and $\lambda_{5}$ as follow [28]:

$$
\begin{aligned}
& \lambda_{4}=\frac{g^{2}}{2 m_{W}^{2}} M_{H^{ \pm}}^{2} \quad, \quad \lambda_{6}=\frac{g^{2}}{2 m_{W}^{2}} M_{A}^{2}, \lambda_{3}=\frac{g^{2}}{8 m_{W}^{2}} \frac{\sin \alpha \cos \alpha}{\sin \beta \cos \beta}\left(M_{H}^{2}-M_{h}^{2}\right)-\frac{\lambda_{5}}{4} \\
& \lambda_{1}=\frac{g^{2}}{8 \cos \beta^{2} m_{W}^{2}}\left[\cos ^{2} \alpha m_{H}^{2}+\sin ^{2} \alpha m_{h}^{2}-\frac{\sin \alpha \cos \alpha}{\tan \beta}\left(m_{H}^{2}-m_{h}^{2}\right)\right]-\frac{\lambda_{5}}{4}\left(-1+\tan ^{2} \beta\right) \\
& \lambda_{2}=\frac{g^{2}}{8 \sin ^{2} \beta m_{W}^{2}}\left[\sin ^{2} \alpha m_{H}^{2}+\cos ^{2} \alpha m_{h}^{2}-\sin \alpha \cos \alpha \tan \beta\left(m_{H}^{2}-m_{h}^{2}\right)\right]+\frac{\lambda_{5}}{4}\left(1-\frac{1}{\tan ^{2} \beta}\right)
\end{aligned}
$$

We are free to take as 7 independent parameters $\left(\lambda_{i}\right)_{i=1, \ldots, 6}$ and $\tan \beta$ or equivalently the four physical scalar masses, $\tan \beta, \alpha$ and one of the $\lambda_{i}$. In what follows we will take as free parameters:

$$
\lambda_{5}, M_{h^{0}}, M_{H^{0}}, M_{A^{0}}, M_{H \pm}, \tan \beta \text { and } \sin \alpha
$$

We list hereafter the Feynman rules in the general 2HDM for the trilinear scalar couplings relevant for our study. They are written in terms of the physical masses, $\alpha, \beta$ and the soft breaking term $\lambda_{5}[28]$ :

$$
\begin{aligned}
H^{0} H^{+} H^{-}= & \frac{-i g}{M_{W} \sin 2 \beta}\left(M_{H^{0}}^{2}\left(\cos \beta^{3} \sin \alpha+\sin \beta^{3} \cos \alpha\right)+M_{H^{ \pm}}^{2} \sin 2 \beta \cos (\beta-\alpha)\right. \\
& \left.-\sin (\beta+\alpha) \lambda_{5} v^{2}\right)
\end{aligned}
$$

\footnotetext{
${ }^{*}$ It exist several ways of writing such a potential see [27] for a discussion
} 


$$
\begin{aligned}
H^{0} H^{+} G^{-}= & \frac{i g}{2 M_{W}} \sin (\beta-\alpha)\left(M_{H^{0}}^{2}-M_{H^{ \pm}}^{2}\right) \\
h^{0} H^{+} H^{-}= & \frac{-i g}{M_{W} \sin 2 \beta}\left(M_{h^{0}}^{2}\left(\cos \alpha \cos \beta^{3}-\sin \alpha \sin \beta^{3}\right)+M_{H^{ \pm}}^{2} \sin 2 \beta \sin (\beta-\alpha)\right. \\
& \left.-\cos (\beta+\alpha) \lambda_{5} v^{2}\right) \\
h^{0} H^{+} G^{-}= & \frac{-i g}{2 M_{W}} \cos (\beta-\alpha)\left(M_{h^{0}}^{2}-M_{H^{ \pm}}^{2}\right) \\
A^{0} H^{+} G^{-}= & \frac{-g}{2 M_{W}}\left(M_{H^{ \pm}}^{2}-M_{A}^{2}\right) \quad, \quad v^{2}=\frac{2 M_{W}^{2}}{g^{2}}
\end{aligned}
$$

We need also the couplings of the scalar bosons to a pair of fermions both in 2HDM-I and 2HDM-II. In these couplings, the relevant terms are as follows:

$$
\begin{aligned}
& h^{0} \bar{t} t \propto M_{t} \frac{\cos \alpha}{\sin \beta} \quad, \quad H^{0} \bar{t} t \propto M_{t} \frac{\sin \alpha}{\sin \beta} \quad, \quad A^{0} \bar{t} t \propto \frac{M_{t}}{\tan \beta} \quad 2 \mathrm{HDM}-\mathrm{I}, \mathrm{II} \\
& h^{0} \bar{b} b \propto M_{b} \frac{\cos \alpha}{\sin \beta} \quad, \quad H^{0} \bar{b} b \propto M_{b} \frac{\sin \alpha}{\sin \beta} \quad, \quad A^{0} \bar{b} b \propto \frac{M_{b}}{\tan \beta} \quad 2 \mathrm{HDM}-\mathrm{I} \\
& h^{0} \bar{b} b \propto M_{b} \frac{\sin \alpha}{\cos \beta} \quad, \quad H^{0} \bar{b} b \propto M_{b} \frac{\cos \alpha}{\cos \beta} \quad, \quad A^{0} \bar{b} b \propto M_{b} \tan \beta \quad 2 \mathrm{HDM}-\text { II } \\
& \left(H^{-} \bar{b} t\right)_{L} \propto \frac{M_{b}}{\tan \beta} \quad, \quad\left(H^{-} \bar{b} t\right)_{R} \propto \frac{M_{t}}{\tan \beta} \quad 2 \mathrm{HDM}-\mathrm{I} \\
& \left(H^{-} \bar{b} t\right)_{L} \propto M_{b} \tan \beta \quad, \quad\left(H^{-} \bar{b} t\right)_{R} \propto \frac{M_{t}}{\tan \beta} \quad 2 \mathrm{HDM}-\mathrm{II}
\end{aligned}
$$

To constrain the scalar sector parameters we will use both vacuum stability conditions as well as tree level unitarity constraints. In our study, we use the vacuum stability conditions from $[29,30]$, which are given by:

$$
\begin{gathered}
\lambda_{1}+\lambda_{3}>0, \quad \lambda_{2}+\lambda_{3}>0, \\
2 \sqrt{\left(\lambda_{1}+\lambda_{3}\right)\left(\lambda_{2}+\lambda_{3}\right)}+2 \lambda_{3}+\lambda_{4}+\min \left(0, \lambda_{5}-\lambda_{4}, \lambda_{6}-\lambda_{4}\right)>0
\end{gathered}
$$

It is well known that the unitarity bounds coming from a tree-level analysis put severe constraints on all scalar trilinear and quartic couplings $[31,32,33]$. The tree level unitarity bounds are derived with the help of the equivalence theorem, which itself is a high-energy approximation where it is assumed that the energy scale is much larger than the $Z^{0}$ and $W^{ \pm}$gauge-boson masses. To derive these unitarity constraints, we consider the following neutral scattering processes

$$
\begin{aligned}
& W_{L}^{+} W_{L}^{-}, Z_{L} Z_{L}, W_{L}^{+} H^{-}, W_{L}^{-} H^{+}, Z_{L} h^{0}, Z_{L} H^{0}, Z_{L} A^{0}, A^{0} A^{0}, H^{+} H^{-}, A^{0} h^{0}, A^{0} H^{0}, \\
& h^{0} h^{0}, h^{0} H^{0}, H^{0} H^{0}
\end{aligned}
$$

as well as the charged channels:

$$
W_{L}^{+} h^{0}, W_{L}^{+} H^{0}, W_{L}^{+} A^{0}, W_{L}^{+} Z_{L}, Z_{L} H^{+}, H^{+} h^{0}, H^{+} H^{0}, H^{+} A^{0}
$$


It has been demonstrated, first in 2HDM with exact discrete symmetry [32] and later in 2HDM with softly broken discrete symmetry [33], that the above neutral scattering processes lead to the following 14 unitarity constraints ${ }^{\dagger}$ :

$$
\left|a_{ \pm}\right|,\left|b_{ \pm}\right|,\left|c_{ \pm}\right|,\left|d_{ \pm}\right|,\left|f_{ \pm}\right|,\left|e_{1,2}\right|,\left|f_{1,2}\right| \leq 16 \pi
$$

with

$$
\begin{aligned}
& a_{ \pm}=3\left(\lambda_{1}+\lambda_{2}+2 \lambda_{3}\right) \pm \sqrt{9\left(\lambda_{1}-\lambda_{2}\right)^{2}+\left(4 \lambda_{3}+\lambda_{4}+0.5\left(\lambda_{5}+\lambda_{6}\right)\right)^{2}} \\
& b_{ \pm}=\lambda_{1}+\lambda_{2}+2 \lambda_{3} \pm \sqrt{\left(\lambda_{1}-\lambda_{2}\right)^{2}+0.25\left(-2 \lambda_{4}+\lambda_{5}+\lambda_{6}\right)^{2}} \\
& c_{ \pm}=\lambda_{1}+\lambda_{2}+2 \lambda_{3} \pm \sqrt{\left(\lambda_{1}-\lambda_{2}\right)^{2}+0.25\left(\lambda_{5}-\lambda_{6}\right)^{2}} \\
& e_{1}=2 \lambda_{3}-\lambda_{4}-\frac{\lambda_{5}}{2}+\frac{5}{2} \lambda_{6} \quad, \quad e_{2}=2 \lambda_{3}+\lambda_{4}-\frac{\lambda_{5}}{2}+\frac{1}{2} \lambda_{6} \\
& f_{+}=2 \lambda_{3}-\lambda_{4}+\frac{5}{2} \lambda_{5}-\frac{1}{2} \lambda_{6} \quad, \quad f_{-}=2 \lambda_{3}+\lambda_{4}+\frac{1}{2} \lambda_{5}-\frac{1}{2} \lambda_{6} \\
& f_{1}=f_{2}=2 \lambda_{3}+\frac{1}{2} \lambda_{5}+\frac{1}{2} \lambda_{6}
\end{aligned}
$$

In addition, it has been noticed in [33], that the inclusion of charged scattering processes leads to one extra unitarity constraint and is given by (see [33] for more details):

$$
p_{1}=2\left(\lambda_{3}+\lambda_{4}\right)-0.5\left(\lambda_{5}+\lambda_{6}\right) \leq 16 \pi
$$

This additional constraint together with the others play an important role in constraining the parameter space of the 2HDM [33]. In our analysis, we take into account also the following constraints when the independent parameters are varied. From the theoretical point of view:

i) The extra contributions to the $\delta \rho$ parameter from the Higgs scalars should not exceed the current limits from precision measurements [34]: $|\delta \rho| \lesssim 0.001$. Such extra contribution to $\delta \rho$ vanishes in the limit $M_{H^{ \pm}}=M_{A}$ which gives $\lambda_{4}=\lambda_{6}$. Under this constraint the $2 \mathrm{HDM}$ scalar potential is $O(4)$ symmetric [35]. In this case $\left(H^{+}, A^{0}, H^{-}\right)$form a triplet under the residual global $S U(2)$ of the Higgs potential. It is this residual symmetry which ensures that $\rho$ is equal to unity at tree level. It is clear from this discussion, that $\delta \rho$ will be violated once the splitting between $H^{ \pm}$and $A^{0}$ is large.

ii) From the requirement of perturbativity for the top and bottom Yukawa couplings, $\tan \beta$ is constrained to lie in the range $0.1 \leq \tan \beta \leq 70$ [36].

iii) It has been shown in [37] that for models of the type 2HDM-II, data on $B \rightarrow X_{s} \gamma$ impose a lower limit of $M_{H^{ \pm}} \gtrsim 350 \mathrm{GeV}$. In type I $2 \mathrm{HDM}$, there is no such constraint on the charged Higgs mass [37]. Recently, it has been shown, using a renormalization group improved calculation of the $B \rightarrow X_{s} \gamma$ branching ratio, that the scale $\Delta=m_{b}-E_{0}$ introduces significant perturbative uncertainties [38]. Such large theory uncertainties may have important implications for searches of new physics in radiative B decays. It is pointed

\footnotetext{
${ }^{\dagger}$ We use the rather conservative unitarity bound $16 \pi$ instead of $8 \pi$
} 

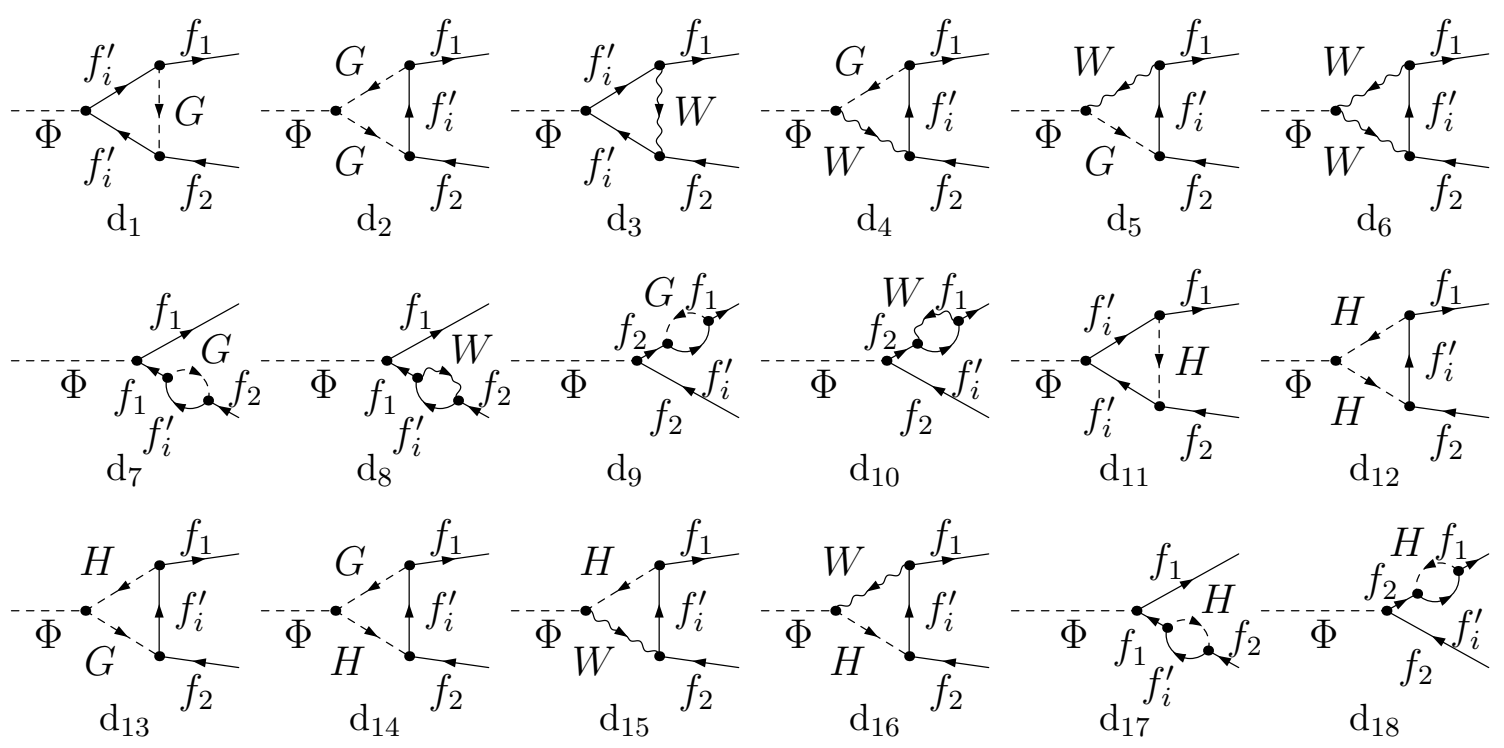

Figure 1: Generic contribution to $\Phi \rightarrow f_{1} f_{2}$ in $\mathrm{SM} d_{1} \rightarrow d_{10}$, in $2 \mathrm{HDM} d_{11} \rightarrow d_{18}$

out in [38], that the lower bound on the charged Higgs mass is reduced to about $200 \mathrm{GeV}$. In our numerical analysis we will ignore these constraints and allow $M_{H \pm} \lesssim 200 \mathrm{GeV}$ in order to localize regions in parameter space where the branching ratios are sizeable.

$i v$ ) The most recent data on the $a_{\mu}=(g-2)_{\mu} / 2$ indicates [39]

$$
\Delta a_{\mu}=a_{\mu}^{\exp }-a_{\mu}^{\mathrm{SM}}=(23.9 \pm 10) \times 10^{-10}
$$

If the SM prediction is based on the hadronic contribution from $e^{+} e^{-}$, the above result (18) shows $2.4 \sigma$ deviation from SM. The dominant $2 \mathrm{HDM}$ contribution to $(g-2)_{\mu}$ come from one loop diagram with Higgs exchange $[40,41]$ and also from two-loop Barr-Zee diagram with heavy fermion running in the upper loop [42, 43]. In both cases, sizeable contribution to $(g-2)_{\mu}$ appear only for relatively light neutral Higgs and large $\tan \beta$. In our study we will assume that all neutral Higgs are heavier than $100 \mathrm{GeV}$ (see below). This assumption make the one loop contribution to $(g-2)_{\mu}$ within experimental range for any value of $\tan \beta \lesssim 70$ and the mixing $\alpha$. The two-loop Barr-Zee diagram has been studied extensively in [43]. It has been shown in [43], that for neutral Higgs bosons heavier than $100 \mathrm{GeV}$ and $\tan \beta$ of the order $60,(g-2)_{\mu}$ two-loop Barr-Zee diagram are within the experimental range. Therefore, since we will assume that all Higgs are heavier than $100 \mathrm{GeV}$ we will ignore $(g-2)_{\mu}$ constraint in what follows.

$v$ ) From the experimental point of view, the combined null-searches from all four CERN LEP collaborations derive the lower limit $M_{H^{ \pm}} \geq 78.6 \mathrm{GeV}(95 \% C L)$, a limit which applies to all models in which $\operatorname{BR}\left(H^{ \pm} \rightarrow \tau \nu_{\tau}\right)+\operatorname{BR}\left(H^{ \pm} \rightarrow c s\right)=1$. For the neutral Higgs bosons, OPAL collaboration has put a limit on $h^{0}$ and $A^{0}$ masses of the 2HDM. They conclude 

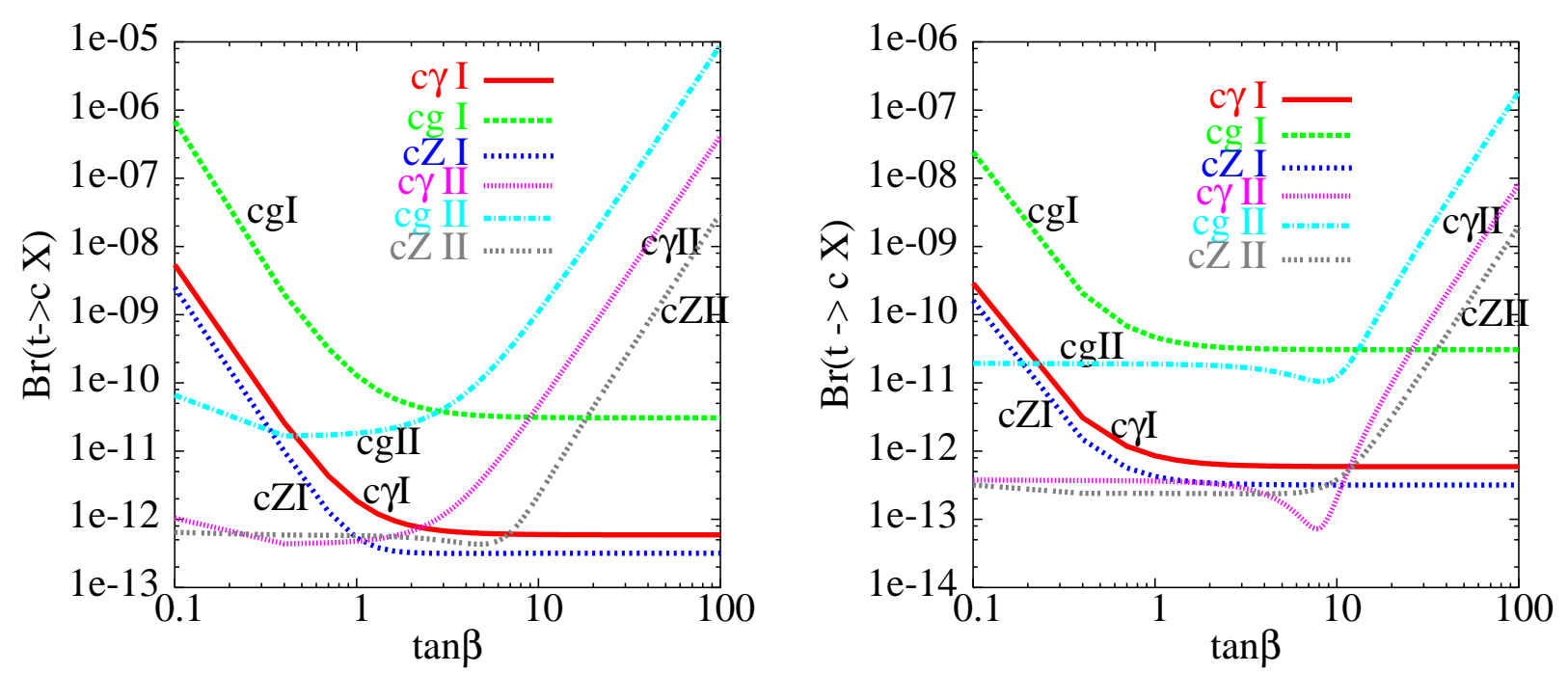

Figure 2: $t \rightarrow c \gamma, t \rightarrow c g$ and $t \rightarrow c Z$ as function of $\tan \beta$ in 2HDM-I and 2HDM-II for $M_{H \pm}=120 \mathrm{GeV}$ (left), $M_{H \pm}=250 \mathrm{GeV}$ (right)

that the regions $1 \lesssim M_{h} \lesssim 44 \mathrm{GeV}$ and $12 \lesssim M_{A} \lesssim 56 \mathrm{GeV}$ are excluded at $95 \% \mathrm{CL}$ independent of $\alpha$ and $\tan \beta$ [44]. In what follows, we will assume that all Higgs masses are greater than $100 \mathrm{GeV}$.

\section{Top FCNC in $2 \mathrm{HDM}$}

In this section, we will discuss the 2HDM contribution to top FCNC couplings $t \rightarrow c \gamma$, $t \rightarrow c g$ and $t \rightarrow c Z$ as well as $t \rightarrow c h^{0}$. In the case of $t \rightarrow c \gamma, t \rightarrow c g$ and $t \rightarrow c Z$ couplings, we reproduce and update the results of ref. [4], while for the case of $t \rightarrow c h^{0}$ couplings, we revisit the study done by S. Béjar et al [14] in the light of the full set of tree level unitarity constraints and vacuum stability conditions.

The full one-loop calculation presented here is done in the 'tHooft gauge with the help of FormCalc [45] and FF packages [46]. Before presenting our numerical results, we would like to mention that we have adopted the following inputs:

$$
\begin{aligned}
& \alpha=1 / 137.035989, M_{W}=80.45, M_{Z}=91.1875 \mathrm{GeV}, s_{W}^{2}=1-M_{W}^{2} / M_{Z}^{2} \quad(19) \\
& M_{t}=174.3, M_{b}=4.7, M_{c}=1.5, M_{s}=0.2 \mathrm{GeV}, V_{c b}=0.04, \alpha_{s}\left(M_{t}\right) \approx 0.105
\end{aligned}
$$

In the 2HDM type I and II, the top FCNC couplings $t \rightarrow c \gamma, t \rightarrow c g$ and $t \rightarrow c Z$ are sensitive only to the charged Higgs mass and $\tan \beta$. We update the plots of ref. [4] for 2 values of charged Higgs mass $M_{H \pm}=120$ and $250 \mathrm{GeV}$ and $\tan \beta \in[0.1,100]$. Our results, in perfect agreement with [4], are illustrated in Fig. (2). In these plots we show the Branching ratios of $t \rightarrow c \gamma, t \rightarrow c g$ and $t \rightarrow c Z$ both in 2HDM-I and 2HDM-II as 
a function of $\tan \beta$ for $M_{H \pm}=120 \mathrm{GeV}$ (left plot) and $M_{H \pm}=250 \mathrm{GeV}$ (right plot). As can be seen from these plots, only at small $\tan \beta$ (for $2 \mathrm{HDM}-\mathrm{I}$ ) and large $\tan \beta$ (for $2 \mathrm{HDM}-\mathrm{II}$ ), can the branching ratios be in the range $10^{-8}-10^{-6}$. Even $t \rightarrow c g$ decay which is proportional to the strong coupling $\alpha_{s}$ does not exceed a branching ratio of $10^{-5}$ at large $\tan \beta \gtrsim 70$. Therefore, if one of those top FCNC emerge at LHC, with a rate larger than $10^{-5}$ that would definitely attest the existence of new sources of flavor violation other than the one induced by the 2HDM structure.

Let us now concentrate on $t \rightarrow c h^{0}$. This top FCNC coupling depends on the scalar parameters through the pure scalar couplings: $h^{0} H^{+} H^{-}$and $h^{0} G^{+} H^{-}$. These scalar couplings eqs. (10), depend on the seven scalar parameters listed above eq. (8) and may be sources of enhancement. Feynman diagrams which depend on these pure scalar couplings are depicted in Fig. (1): $d_{12}, d_{13}$ and $d_{14}$. Top FCNC $t \rightarrow c h^{0}$ depends also on the bottom Yukawa coupling through $H^{+} \bar{b} t$ and/or $h^{0} b \bar{b}$ interaction which could also enhance the width of $t \rightarrow c h^{0}$ for large $\tan \beta$. These kinds of diagrams can be seen in $d_{1,3}$ and $d_{11 \rightarrow 18}$.

At large $\tan \beta$, it is expected that the main enhancement comes from diagrams of the type $d_{11}$ and $d_{12}$. If we neglect the charm mass and right coupling $\left(H^{-} \bar{f} t\right)_{R} \propto 1 / \tan \beta$, which are suppressed for large $\tan \beta$, the amplitudes $M_{11}$ of $d_{11}$ and $M_{12}$ of $d_{12}$ are given by:

$$
\begin{aligned}
& M_{11} \approx \frac{h^{0} f \bar{f}}{16 \pi^{2}} M_{t} V_{f t} V_{c f}^{*} \quad\left(H^{-} \bar{f} t\right)_{L} \quad\left(H^{-} \bar{f} c\right)_{L} \quad f_{1}\left(M_{H \pm}^{2}, M_{t}^{2}, M_{f}^{2}, M_{h}^{2}\right) v_{t} \frac{1+\gamma_{5}}{2} u_{c} \\
& M_{12} \approx \frac{h^{0} H^{+} H^{-}}{16 \pi^{2}} M_{t} V_{f t} V_{c f}^{*} \quad\left(H^{-} \bar{f} t\right)_{L} \quad\left(H^{-} \bar{f} c\right)_{L} \quad f_{2}\left(M_{H \pm}^{2}, M_{t}^{2}, M_{f}^{2}, M_{h}^{2}\right) v_{t} \frac{1+\gamma_{5}}{2} u(21)
\end{aligned}
$$

$f_{1}$ and $f_{2}$ are form factors and $f$ is the internal quark. The couplings $h^{0} f \bar{f}, h^{0} H^{+} H^{-}$and $\left(H^{-} \bar{f} t\right)_{L}$ are given in eqs. $(10,11)$. Since in $2 \mathrm{HDM}-\mathrm{II}, h^{0} f \bar{f}$ behaves like $m_{f} \tan \beta$ at large $\tan \beta$ and the coupling $h^{0} H^{+} H^{-}$is enhanced for large $\tan \beta$ (see discussion below), one can expect that the amplitude of those diagrams grows like $\tan ^{3} \beta$. But care has to be taken, since the $\tan ^{3} \beta$ enhancement of the amplitude may be reduced by the CKM elements, such as $V_{c b}$, as well as by the GIM cancellation. When computing the branching ratio $B r\left(t \rightarrow c h^{0}\right)$, in the top width we include both the tree level decay $t \rightarrow W^{+} b$ as well as $t \rightarrow H^{+} b$ when it is open. We make a comparison of our results with the results obtained by Béjar et al [14], and find perfect agreement. However the parameters set chosen by Ref. [14] to illustrate their results violate both unitarity and perturbativity constraints on $\lambda_{i}[32,33]$.

We have performed a systematic scan over the full parameter in the range allowed by the theoretical constraints such as unitarity constraints and vacuum stability conditions as well as by the experimental data. As one can see from the scatter plot Fig. (4), the branching ratio of $t \rightarrow c h^{0}$ remains less than $8 \times 10^{-5}$ for light charged Higgs mass and large $\tan \beta$.

Let us start by discussing the order of magnitude of the triple coupling $h^{0} H^{+} H^{-}$. In the left plot of Fig. (3) we illustrate the ratio $R=\left|h^{0} H^{+} H^{-} / h^{0} h^{0} h^{0}\right|$, with $h^{0} h^{0} h^{0}$ being the SM coupling given by $h^{0} h^{0} h^{0}=3 g M_{h^{0}}^{2} /\left(2 M_{W}\right)$. In this plot, we use $M_{h^{0}}=125 \mathrm{GeV}$ and the other parameters take the values $M_{H \pm}=150, M_{A^{0}}=215$ and $M_{H^{0}}=250 \mathrm{GeV}$. As it can be seen from this plot, unitarity constraints allow $\tan \beta$ to be as large as 70 

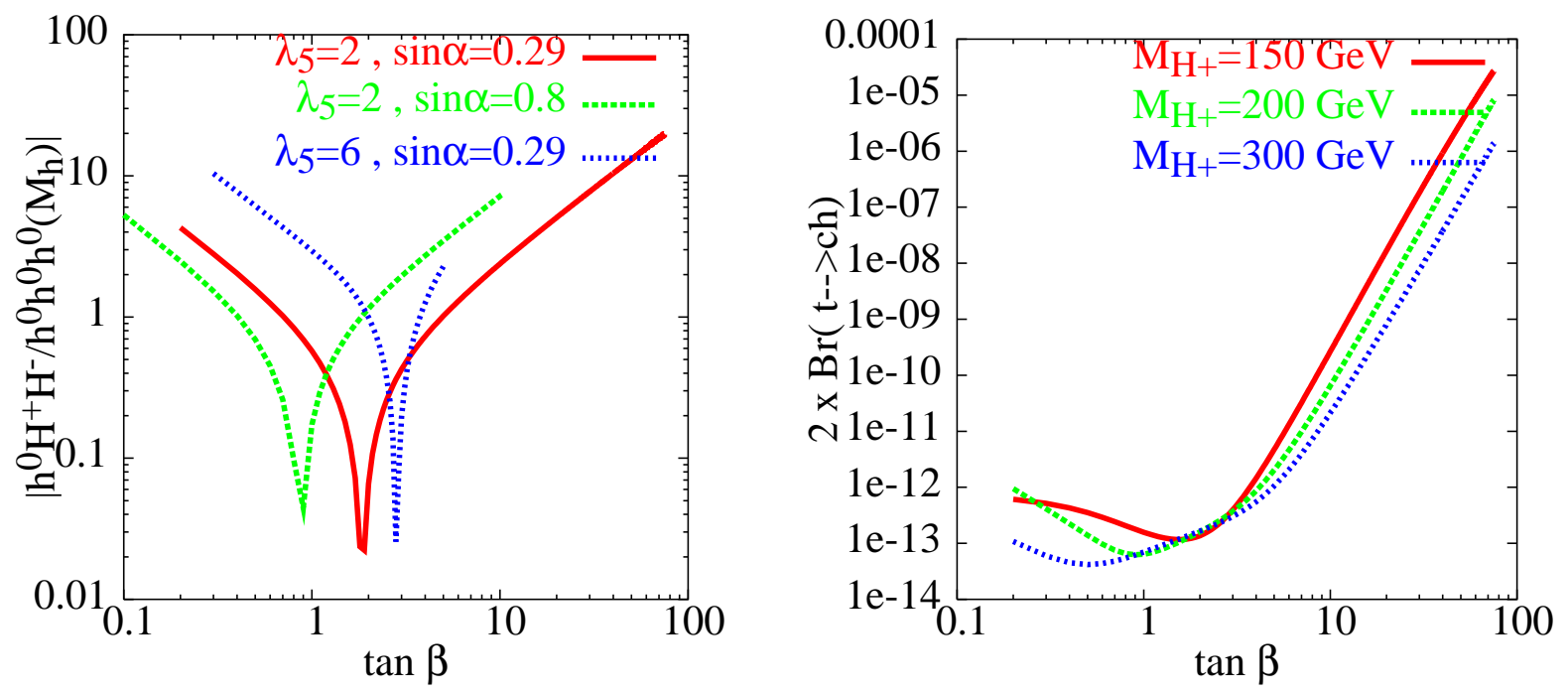

Figure 3: Left, Absolute value of $h^{0} H^{+} H^{-}$coupling normalized to SM coupling $h^{0} h^{0} h^{0}$ taken at $M_{h}^{0}=125 \mathrm{GeV}$, the other parameters are fixed as $M_{H \pm}=150, M_{A^{0}}=215$ and $M_{H^{0}}=250 \mathrm{GeV}, \lambda_{5}=2$. Right, $2 \times B r\left(t \rightarrow c h^{0}\right)$ in $2 \mathrm{HDM}-\mathrm{II}$ as function of $\tan \beta$ for several values of $M_{H \pm}$ and $M_{h^{0}}=125, M_{A^{0}}=215, M_{H^{0}}=250 \mathrm{GeV}, \lambda_{5}=2$ and $\sin \alpha=0.29$
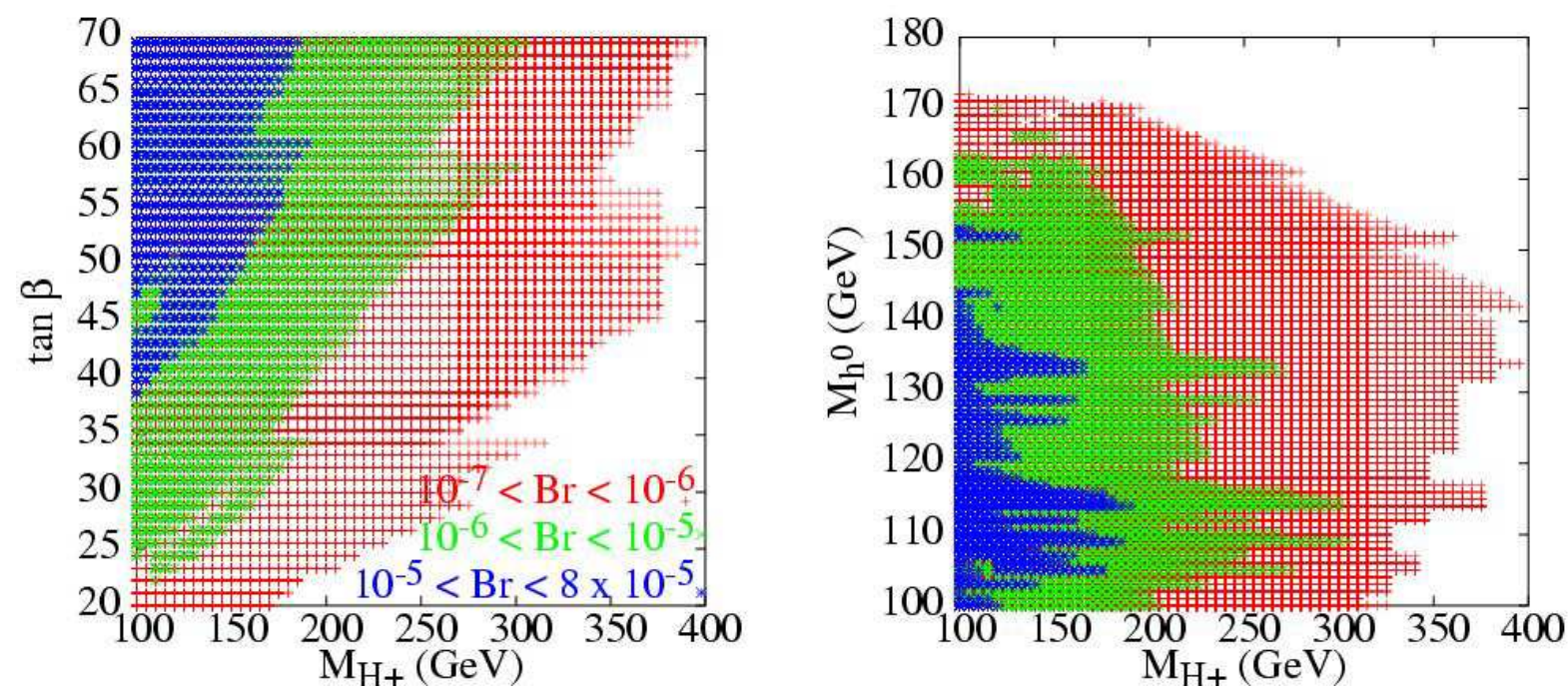

Figure 4: Scatter plots for $\operatorname{Br}\left(t \rightarrow c h^{0}\right)$ in the $\left(\tan \beta, M_{H \pm}\right)$ plan (left) and $\left(M_{h^{0}}, M_{H \pm}\right)$ plan. We scan over: $100 \lesssim M_{h^{0}} \lesssim 175,100 \lesssim M_{H^{0}, A^{0}, H \pm} \lesssim 600 \mathrm{GeV},-1 \lesssim \sin \alpha \lesssim 1$ and $20 \lesssim \tan \beta \lesssim 70$ and $\lambda_{5}=M_{A}^{2} / v^{2}$.

only for $\sin \alpha \approx 0.29$ and $\lambda_{5}=2$. For large $\sin \alpha=0.8$ unitarity already breaks down for $\tan \beta \approx 10$. It is clear that the coupling $h^{0} H^{+} H^{-}$reach its maximal value for large $\tan \beta$. The minimum observed in the $h^{0} H^{+} H^{-}$coupling is due to a cancellation between 
the different terms which contribute to $h^{0} H^{+} H^{-}$(eq.(9)). Such a minimum depends of course on the values of $\lambda_{5}$ and $\sin \alpha$. Since the coupling $h^{0} H^{+} H^{-}$depends linearly on $\lambda_{5}$, it is obvious that this coupling gets its largest value for the largest value of $\lambda_{5}$ tolerated by perturbativity: $\lambda_{5} \approx 8 \pi$. However, unitarity requires that for large $\lambda_{5}$, large $\tan \beta$ is not allowed (see left plot Fig. 3 for $\lambda_{5}=6$ ).

Having the order of magnitude of $h^{0} H^{+} H^{-}$in mind, we show in the right panel of Fig. (3) the Branching ratio of $t \rightarrow c h^{0}$ as a function of $\tan \beta$ for several values of $M_{H \pm}$ with $M_{h^{0}}=125, M_{A^{0}}=215, M_{H^{0}}=250 \mathrm{GeV}, \lambda_{5}=2$ and $\sin \alpha=0.29$. For large $\tan \beta$ and light charged Higgs $M_{H \pm}=150 \mathrm{GeV}, \operatorname{Br}\left(t \rightarrow c h^{0}\right)$ can reach value larger than $10^{-5}$ while for $M_{H \pm}=200 \mathrm{GeV}$, the $\operatorname{Br}\left(t \rightarrow c h^{0}\right)$ is already less than $10^{-5}$ even for large $\tan \beta \approx 70$. This enhancement is mainly attributed to the triple scalar coupling $h^{0} H^{+} H^{-}$as well as to the bottom Yukawa coupling which are both enhanced for large $\tan \beta$ as one can see from eqs. $(20,21)$.

We also provide scatter plots Fig. (4) for $\operatorname{Br}\left(t \rightarrow c h^{0}\right)$ in $\left(M_{H \pm}, \tan \beta\right)$ and $\left(M_{H \pm}, M_{h^{0}}\right)$ plans. There is only a small window for large $\tan \beta$ and $M_{H \pm} \lesssim 180 \mathrm{GeV}$ where $\operatorname{Br}\left(t \rightarrow c h^{0}\right)$ can be larger than $10^{-5}$. As argued before, due to the large theoretical uncertainties in the $B \rightarrow X_{s} \gamma$ calculation, the lower bound on the charged Higgs mass is reduced to about $200 \mathrm{GeV}$ [38]. From the left panel of Fig. 4, it is illustrated that for charged Higgs mass $M_{H \pm} \in[200,300] \mathrm{GeV}$ and large $\tan \beta, \operatorname{Br}\left(t \rightarrow c h^{0}\right) \in\left[10^{-6}, 10^{-5}\right]$. One concludes that the top FCNC $t \rightarrow c h^{0}$ in $2 \mathrm{HDM}$ can reach a detectable rate at LHC with the high luminosity option.

\section{Higgs FCNC in 2HDM}

\subsection{Higgs FCNC in SM}

Before presenting our results in $2 \mathrm{HDM}$, we would like to comment on the Branching ratio of $H \rightarrow \bar{t} c$ in the SM. Recently, an estimation using dimensional analysis and power counting, appeared both for $\operatorname{Br}(H \rightarrow \bar{s} b)$ [47] and $\operatorname{Br}(H \rightarrow \bar{t} c)$ [15] while in [16], the full diagrammatic calculation has been done. Here we comment on results for $\operatorname{Br}(H \rightarrow \bar{t} c)$ and $\operatorname{Br}(H \rightarrow \bar{b} s)[16]$.

As expected the branching ratios of these decays are very suppressed due to GIM mechanism $[15,16,47]$. In the case of $H \rightarrow \bar{t} c$, the internal fermions are bottom, strange and down quarks with masses close to degeneracy. The GIM suppression in this case is very severe, and the $\mathrm{Br}$ is of the order $10^{-13}$. For $H \rightarrow \bar{b}$ s the internal fermions are top, charm and up quarks and with the large top mass as well as large splitting between the internal quarks, the Branching ratio of $H \rightarrow \bar{b} s$ is in the range $10^{-9}-10^{-7} . H \rightarrow \bar{b} s$ has another advantage over $H \rightarrow \bar{t} c$ which is the fact that $H \rightarrow \bar{b} s$ can be open for Higgs mass $M_{H}<2 M_{W}$ and hence the decay width of the Higgs can be very narrow which can enhance the Branching ratio for $M_{H} \lesssim 2 M_{W}$. The decay $H^{0} \rightarrow \bar{t} c$ is open only when $M_{H}>m_{t}+m_{c} \approx 2 M_{Z}$. For a Higgs mass in this range, the decay channels $H^{0} \rightarrow\left\{W^{+} W^{-}, Z^{0} Z^{0}\right\}$ are already open and so the total width of the Higgs is no longer narrow. Such a large decay width may reduce the Branching ratio of $H^{0} \rightarrow \bar{t} c$. For instance, for Higgs mass heavier than 250 
$\mathrm{GeV}$, we get a branching ratio of the order $10^{-14} \rightarrow 10^{-12}$ for $H \rightarrow \bar{t} c$. New sources of flavor violation like charged Higgs are expected to enhance the branching ratio of $\Phi \rightarrow \bar{t} c$.

\section{$4.2 \Phi \rightarrow \bar{t} c$}

In this section we discuss the 2HDM contributions to the Higgs FCNC. We concentrate mainly on $h^{0} \rightarrow \bar{t} c, H^{0} \rightarrow \bar{t} c$ and $A^{0} \rightarrow \bar{t} c$. The generic Feynman diagrams which contribute to those FCNC are shown in fig .(1). As we can see, the additional interactions like $\Phi^{0} H^{+} H^{-}, \Phi^{0} H^{+} G^{-}$and $\Phi^{0} H^{+} W^{-}$give new contributions to $\Phi^{0} \rightarrow \bar{t} c$.

The calculation of $h^{0} \rightarrow \bar{t} c, H^{0} \rightarrow \bar{t} c$ and $A^{0} \rightarrow \bar{t} c$ in 2HDM has been first done in Ref. [15]. In Ref. [15], only trilinear Higgs self-couplings $\lambda_{H H H}$ have been constrained by the maximum unitarity limit tolerated for the SM trilinear coupling:

$$
\left|\lambda_{H H H}\right| \leq\left|\lambda_{H H H}^{S M}\left(M_{H}=1 \mathrm{TeV}\right)\right|=\frac{3 g(1 \mathrm{TeV})^{2}}{2 M_{W}}
$$

We have checked that the above constraints (22) are not enough to guarantee unitarity and perturbativity constraints. For instance, for the parameter set considered in Ref. [15], $\lambda_{1}$ can take extremely large values of the order $\approx 5000$ which is not tolerated neither by perturbativity nor by unitarity constraints. We perform a cross check with Ref. [15], and our results ${ }^{\ddagger}$ are about 3 times larger than those presented in Ref. [15].

The decay width $\Gamma_{\Phi}$ of all scalar particles $h^{0}, H^{0}$ and $A^{0}$ are computed at tree level in 2HDM as follows:

$$
\Gamma_{\Phi}=\sum_{f} \Gamma(\Phi \rightarrow f \bar{f})+\Gamma(\Phi \rightarrow V V)+\Gamma\left(\Phi \rightarrow V H_{i}\right)+\Gamma\left(\Phi \rightarrow H_{i} H_{j}\right)
$$

QCD corrections to $\Phi \rightarrow f \bar{f}$ and $\Phi \rightarrow\left\{g g, \gamma \gamma, \gamma Z, V^{*} V^{*}, V V^{*}, V^{*} H_{i}\right\}$ decays are not included in the width. Since the width of $\Phi \rightarrow \bar{t} c$ does not become comparable to $\Phi \rightarrow$ $\{g g, \gamma \gamma, \gamma Z\}$, it is not necessary to include them in the computation. The full width of the Higgs bosons is taken from [48] without QCD corrections. As stated before, we perform a systematic scan over the 2HDM parameter space using unitarity constraints and vacuum stability conditions on the scalar sector parameters. In our numerical analysis, we limit ourself to 2HDM type II. As shown in Ref. [15], the rates in 2HDM-I are smaller than the rates in 2HDM-II. We also present numerics for CP even Higgs only $\Phi=h^{0}, H^{0}$ and comment on our finding for $A^{0} \rightarrow \bar{t} c$ in 2HDM type I and II.

$h^{0} \rightarrow \bar{t} c$

We illustrate first in Fig. (5) the branching ratio of $h^{0} \rightarrow \bar{t} c$ in 2HDM-II as a function of $M_{h^{0}}$ (left) and $\tan \beta$ (right). The parameters are fixed as: $M_{A^{0}}=215, M_{H^{0}}=250 \mathrm{GeV}$, $\lambda_{5}=2$ and $\sin \alpha=0.5$ and $M_{H \pm}=150 \mathrm{GeV}$ (left) and $M_{h^{0}}=220 \mathrm{GeV}$ (right). In both plots (left and right), one can see that the Branching ratio of $h^{0} \rightarrow \bar{t} c$, like the $\operatorname{Br}\left(t \rightarrow c h^{0}\right)$,

\footnotetext{
${ }^{\ddagger}$ We reproduce our results by 2 different and independent programs.
} 

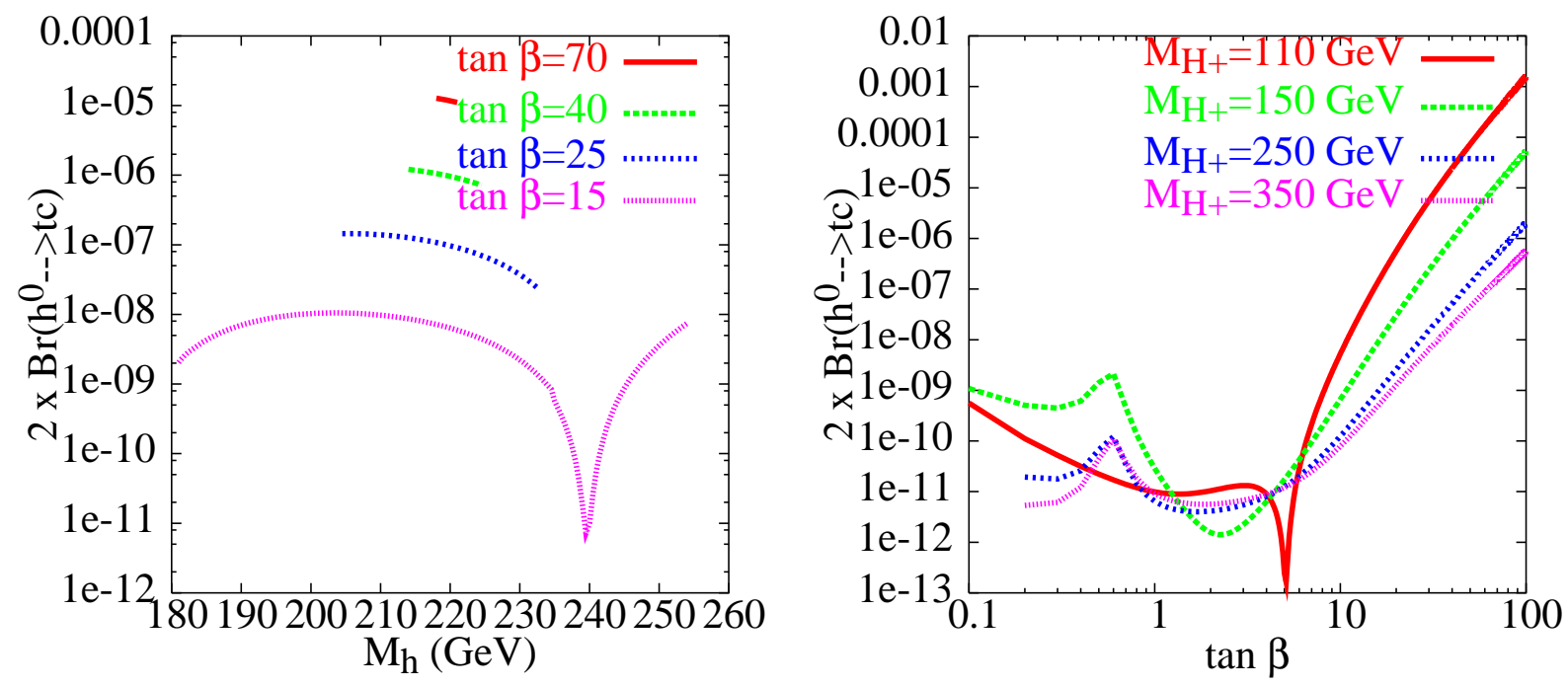

Figure 5: $2 \times \operatorname{Br}\left(h^{0} \rightarrow \bar{t} c\right)$ in 2HDM-II as a function of $M_{h}$ (left) and $\tan \beta$ (right) with $M_{A^{0}}=215, M_{H^{0}}=250 \mathrm{GeV}, \lambda_{5}=2$ and $\sin \alpha=0.5$ and $M_{H \pm}=150 \mathrm{GeV}$ (left) and $M_{h^{0}}=220 \mathrm{GeV}($ right $)$
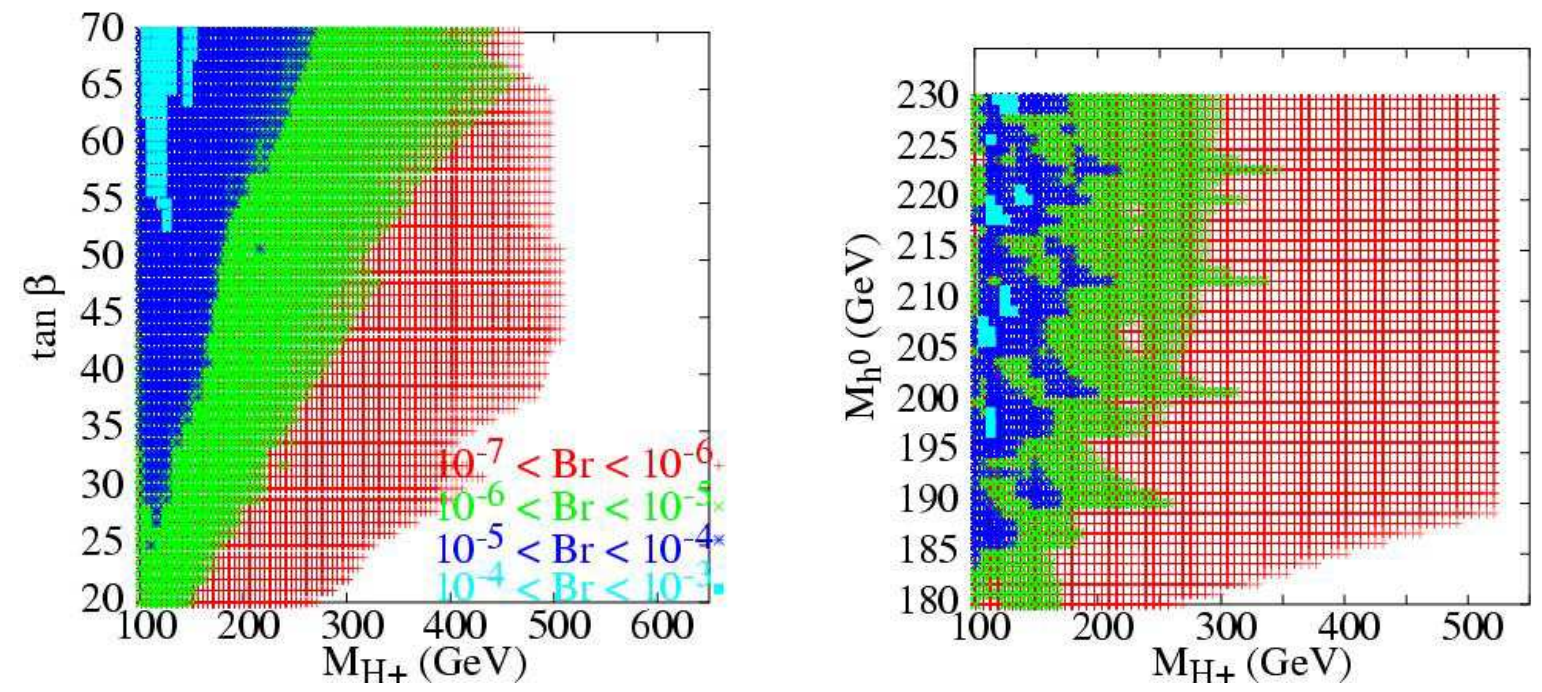

Figure 6: Scatter plots for $2 \times \operatorname{Br}\left(h^{0} \rightarrow \bar{t} c\right)$ in the $\left(\tan \beta, M_{H \pm}\right)$ (left) and $\left(M_{h^{0}}, M_{H \pm}\right)$ plans. We scan over: $180 \lesssim M_{h^{0}} \lesssim 230,190 \lesssim M_{H^{0}} \lesssim 230100 \lesssim M_{A^{0}, H \pm} \lesssim 600 \mathrm{GeV}$, $-1 \lesssim \sin \alpha \lesssim 1$ and $20 \lesssim \tan \beta \lesssim 70$ and $\lambda_{5}=M_{A}^{2} / v^{2}$.

can be of the order $10^{-5}$ only for large $\tan \beta$ and relatively light charged Higgs mass. As in the case of $t \rightarrow c h^{0}$, this enhancement at large $\tan \beta$ is due to bottom Yukawa coupling as well as to the triple scalar coupling $h^{0} H^{+} H^{-}$.

We show on the right panel of Fig. (5) the Branching ratio of $h^{0} \rightarrow \bar{t} c$ as a function of 

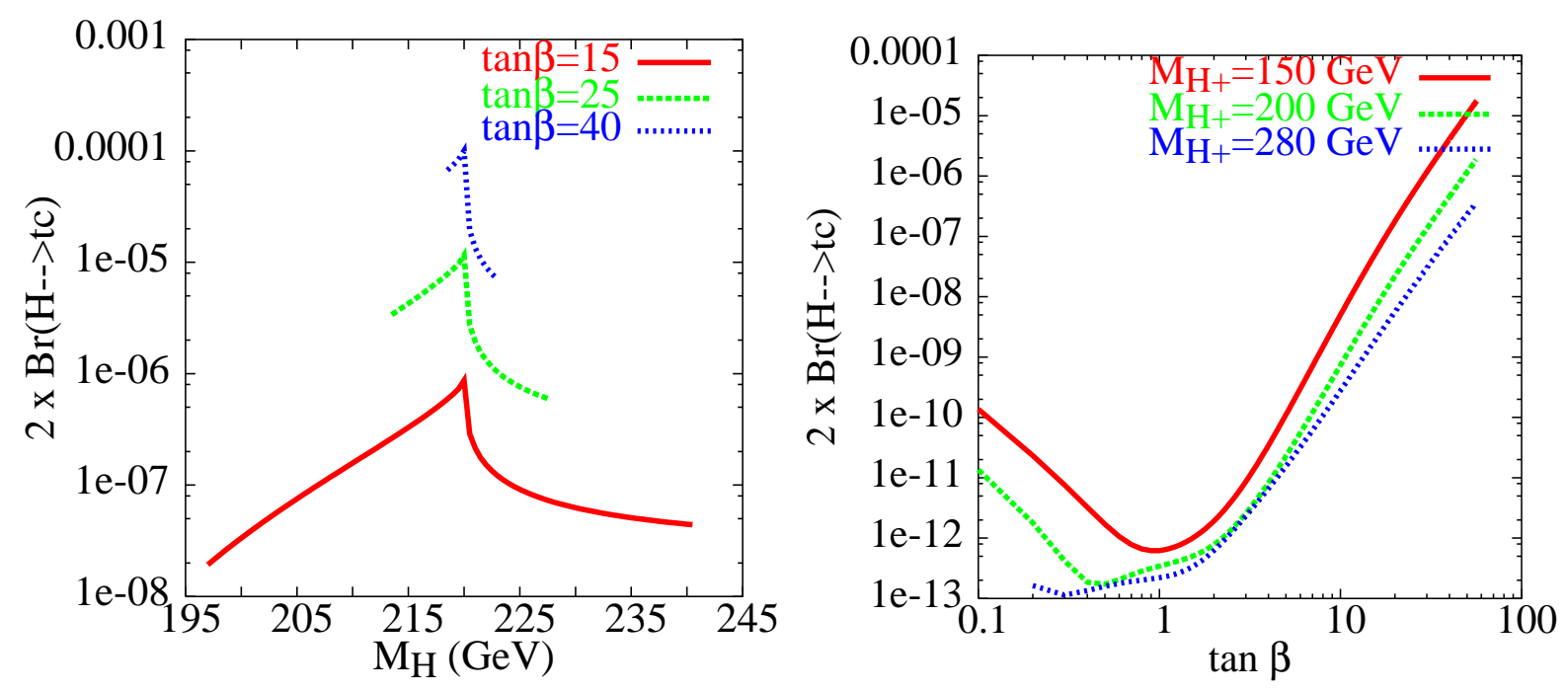

Figure 7: Branching ratio for $2 \times B r\left(H^{0} \rightarrow \bar{t} c\right)$ as a function of $M_{H^{0}}$ (left) and tan $\beta$ (right) in 2HDM-II $M_{h^{0}}=120$, and $M_{A^{0}}=200 \mathrm{GeV}, \lambda_{5}=1$, sin $\alpha=0.75$ with $M_{H \pm}=110 \mathrm{GeV}$ for left and $M_{H^{0}}=220 \mathrm{GeV}$ for right panel

$\tan \beta$ for several values of charged Higgs mass $M_{H \pm}$. The maximum rate for $\operatorname{Br}\left(h^{0} \rightarrow\right.$ $\bar{t} c) \approx 4 \times 10^{-4}$ is obtained for $M_{H \pm}=110 \mathrm{GeV}$ and large $\tan \beta \approx 65$. The reason for this enhancement is due to threshold effect, since at $M_{h^{0}}=220 \mathrm{GeV}$ and $M_{H \pm}=110 \mathrm{GeV}$ the decay channel $h^{0} \rightarrow H^{+} H^{-}$is open ${ }^{\S}$. For charged Higgs mass away from the threshold region, the $\operatorname{Br}\left(h^{0} \rightarrow \bar{t} c\right)$ is reduced and for $M_{H \pm}=150 \mathrm{GeV}$, it is of the order $10^{-5}$ at $\tan \beta \approx 70$.

In the scatter plots Fig. (6) it is illustrated in the left panel that for large $\tan \beta \gtrsim 30$ and light charged Higgs $M_{H \pm} \lesssim 250 \mathrm{GeV}$ the $\operatorname{Br}\left(h^{0} \rightarrow \bar{t} c\right) \in\left[10^{-5}, 10^{-3}\right]$. The maximum branching ratio is obtained close to threshold region where the decay $h^{0} \rightarrow H^{+} H^{-}$is open. For charged Higgs mass $M_{H \pm} \gtrsim 250 \mathrm{GeV}, \operatorname{Br}\left(h^{0} \rightarrow \bar{t} c\right) \lesssim 10^{-5}$ for all range of tan $\beta$. From the right panel, one can see that the $\operatorname{Br}\left(h^{0} \rightarrow \bar{t} c\right)$ is not very sensitive to the CP-even mass $M_{h^{0}}$.

$H^{0} \rightarrow \bar{t} c$

We now discuss the 2HDM contribution to $H^{0} \rightarrow \bar{t} c$. We perform a scan in the 2HDM parameter space and find a region where the branching ratio is sizeable. The conclusion is similar to $h^{0} \rightarrow \bar{t} c$. To have a large branching ratio we need to maximize the coupling $H^{0} H^{+} H^{-}$and the bottom Yukawa coupling. This is achieved by taking large tan $\beta$.

In the left panel of Fig. (7), we illustrate the Branching ratio of $H^{0} \rightarrow \bar{t} c$ as a function of CP even Higgs mass $M_{H}$ for several values of $\tan \beta$. As it can be seen, for moderate $\tan \beta<15$, the Branching ratio is less than $10^{-6}$. When the $\mathrm{CP}$ even mass $M_{H^{0}} \approx 2 M_{H \pm} \approx 220 \mathrm{GeV}$

\footnotetext{
${ }^{\S}$ see next plot for similar threshold effect for $H^{0} \rightarrow \bar{t} c$
} 

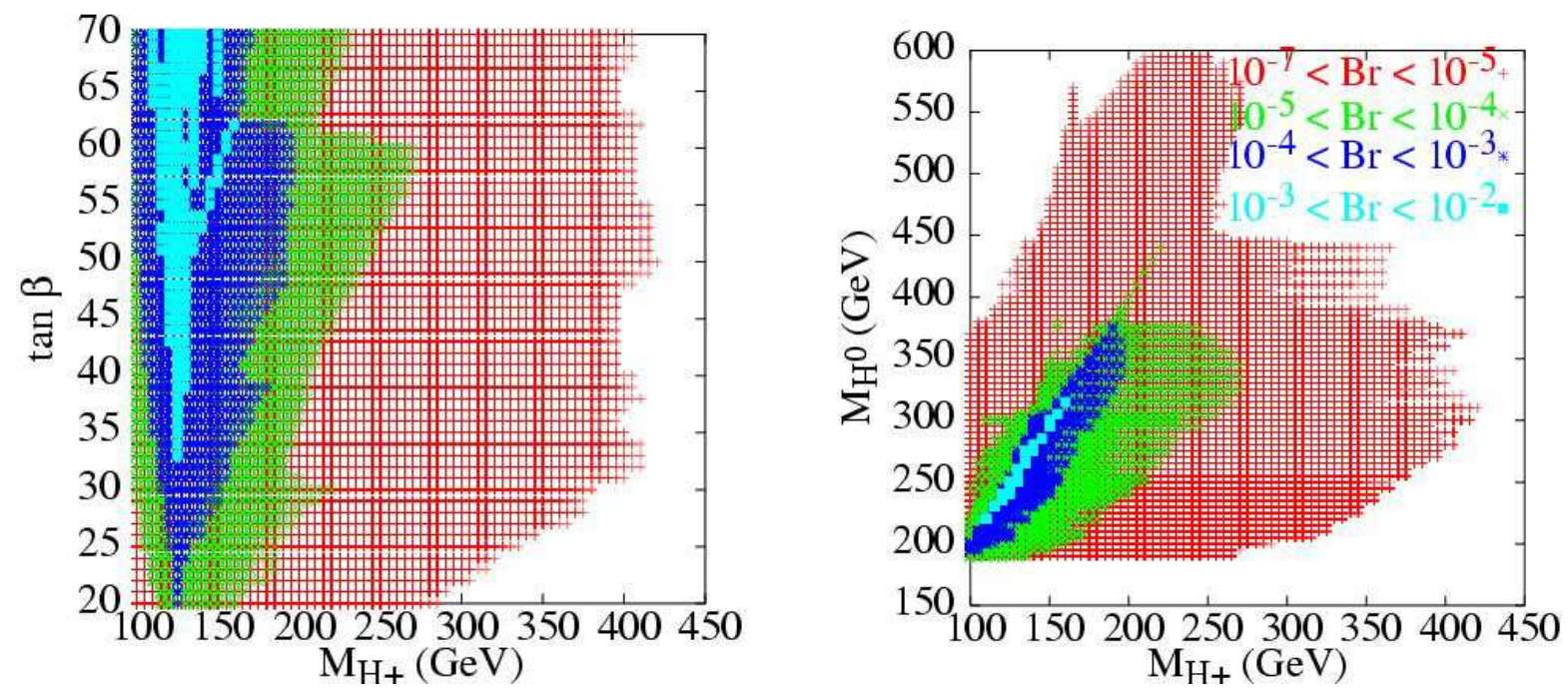

Figure 8: Scatter plots for $2 \times \operatorname{Br}\left(H^{0} \rightarrow \bar{t} c\right)$ in the $\left(\tan \beta, M_{H \pm}\right)$ (left) and $\left(M_{H^{0}}, M_{H \pm}\right)$ plans. We scan over: $100 \lesssim M_{h^{0}} \lesssim 230,180 \lesssim M_{H^{0}} \lesssim 600100 \lesssim M_{A^{0}, H \pm} \lesssim 600 \mathrm{GeV}$, $-1 \lesssim \sin \alpha \lesssim 1$ and $20 \lesssim \tan \beta \lesssim 70$ and $\lambda_{5}=M_{A}^{2} / v^{2}$.

one can see a peak. Such a peak corresponds to the opening of the decay channel $H^{0} \rightarrow$ $H^{+} H^{-}$. This threshold effect is also amplified by the fact that the coupling $H^{0} H^{+} H^{-}$ takes its maximal value for $M_{H^{0}} \approx 220 \mathrm{GeV}$ and large $\tan \beta$. In this region, the Branching ratio can reach $3 \times 10^{-4}$ at large $\tan \beta \approx 50$. As can be seen in the left plot, for large $\tan \beta \geq 25$ unitarity is violated apart from a small region around $M_{H} \approx 220 \mathrm{GeV}$. Once the Higgs mass $M_{H}$ becomes larger than $2 M_{H \pm}$, the decays $H^{0} \rightarrow\left\{H^{+} H^{-}, h^{0} h^{0}\right\}$ are open, the width $\Gamma_{H^{0}}$ becomes large and hence the Branching ratio is reduced.

In the right panel of Fig. (7), we show the branching ratio for $H^{0} \rightarrow \bar{t} c$ as a function of $\tan \beta$ for several values of $M_{H \pm}$. As for the $h^{0} \rightarrow \bar{t} c$ case, the branching ratio is enhanced by large $\tan \beta$ and rather light charged Higgs mass. Also in this case, for large $\tan \beta$ the coupling $H^{0} H^{+} H^{-}$reaches its maximal value. It is clear from the right plot that the Branching ratio $\operatorname{Br}\left(H^{0} \rightarrow \bar{t} c\right)$ can be greater than $10^{-5}$ only for large $\tan \beta \gtrsim 45$ and $M_{H \pm}=150 \mathrm{GeV}$. Once the charged Higgs mass becomes large, the $\operatorname{Br}\left(H^{0} \rightarrow \bar{t} c\right)$ decreases. This can be seen in the right plot of Fig. (7) where $M_{H \pm}=250 \mathrm{GeV}$, the $\operatorname{Br}\left(H^{0} \rightarrow \bar{t} c\right)$ is less than $10^{-6}$.

We have also performed a systematic scan for this case, and the results are illustrated in Fig. 8. In those scatter plots, we show $\operatorname{Br}\left(H^{0} \rightarrow \bar{t} c\right)$ as function of $\left(M_{H \pm}, \tan \beta\right)$ (left) and $\left(M_{H \pm}, M_{H^{0}}\right)$ (right). Again, close to the threshold region where $H^{0} \rightarrow H^{+} H^{-}$is open, the $\operatorname{Br}\left(H^{0} \rightarrow \bar{t} c\right)$ can be larger than $10^{-3}$ (see left panel of Fig. 8). Such an enhancement requires of course $M_{H \pm} \lesssim 150 \mathrm{GeV}$ and large $\tan \beta$. In this case also, there is a region in which the charged Higgs mass is larger than $200 \mathrm{GeV}$, which could accommodate $B \rightarrow X_{s} \gamma$, and the $\operatorname{Br}\left(H^{0} \rightarrow \bar{t} c\right) \in\left[10^{-5}, 10^{-4}\right]$. From the right panel of Fig. 8, one can see that once the $\bar{t} t$ threshold has been passed $M_{H} \gtrsim 350 \mathrm{GeV}$, the Branching ratio $\operatorname{Br}\left(H^{0} \rightarrow \bar{t} c\right)$ is 


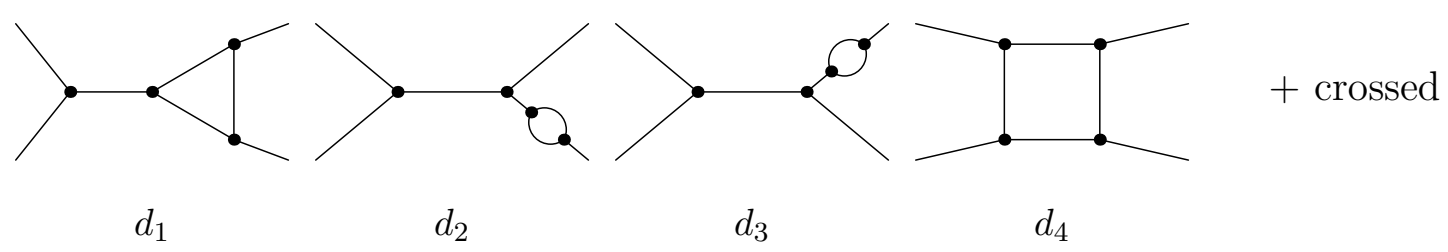

Figure 9: Topological contributions to $e^{+} e^{-} \rightarrow \bar{t} c$ and $\mu^{+} \mu^{-} \rightarrow \bar{t} c$ in $2 \mathrm{HDM}$

reduced.

$A^{0} \rightarrow \bar{t} c$

At the end of this section, we would like to comment on $A^{0} \rightarrow \bar{t} c$ decay in 2HDM type I and II. In $2 \mathrm{HDM}-\mathrm{II}$, we perform a systematic scan over the $2 \mathrm{HDM}$ parameters. It is found that the Branching ratio of $A^{0} \rightarrow \bar{t} c$ decay never exceeds $10^{-8}\left(\right.$ resp $3 \times 10^{-9}$ ) for small (resp large) $\tan \beta$. The reason is that, unlike $h^{0}$ and $H^{0}$, the CP-odd $A^{0}$, being pseudo-scalar, does not couple to a pair of charged Higgs. Therefore $A^{0} \rightarrow \bar{t} c$, has only a unique source of enhancement which is the bottom Yukawa coupling.

In $2 \mathrm{HDM}-\mathrm{I}$, there is a small region for $100<M_{H \pm}<200 \mathrm{GeV}, 176<M_{A^{0}}<2 M_{t} \approx 350$ $\mathrm{GeV}$ and small $0.1<\tan \beta<0.3$ where the Branching ratio of $A^{0} \rightarrow \bar{t} c$ is in the range: $\left[10^{-9}, 3 \times 10^{-7}\right]$. At large $\tan \beta$ the branching ratio decreases quickly and is less than $10^{-14}$ for $\tan \beta>2$. The origin of this is the fact that the couplings $A^{0} b \bar{b}$ and $H^{-} t \bar{b}$ are proportional to $1 / \tan \beta$ and so suppressed for large $\tan \beta$.

\section{Top-charm production at $e^{+} e^{-}, \gamma \gamma$ and muon collid- ers}

There have been several studies looking for collider signatures of the top and Higgs FCNC couplings both at lepton colliders [49] as well as at hadron colliders [50]. Because of the very large mass of the top, Top-charm production at leptons and hadron colliders would have a clear signature.

At LHC, the production and subsequent decay of the neutral CP-even Higgs mediated by FCNC have been analyzed in [15] for 2HDM and [50] for MSSM. It has been demonstrated in those studies that the production rates for the $\bar{b} s+b \bar{s}$ channel can be large while the rates for $\bar{t} c+t \bar{c}$ channel are more modest. Detection for the former channel at LHC is difficult but it is easier for the later [50].

Associate Top-charm production at $e^{+} e^{-}$colliders has been studied in SM [51] and found to be very small. New physics contribution to $e^{+} e^{-} \rightarrow \bar{t} c$ such as SUSY can enhance the cross sections by several orders of magnitude with respect to SM [52]. The top-charm production at lepton colliders $e^{+} e^{-}$and $\mu^{+} \mu^{-}$has been studied in two Higgs doublet models without 


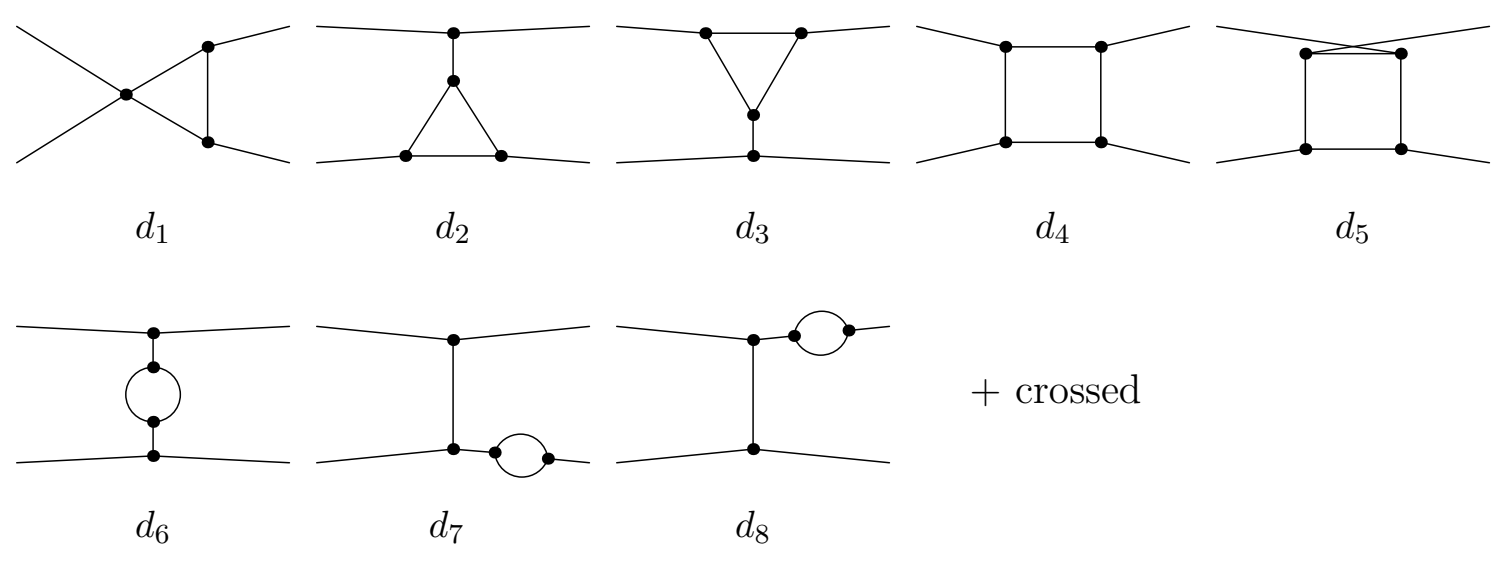

Figure 10: Topological contributions to $\gamma \gamma \rightarrow \bar{t} c$ in $2 \mathrm{HDM}$

Natural Flavor Conservation 2HDM-III which can lead to measurable effects [49, 53]. It has been pointed out that the tree level vertex $\Phi \bar{t} c$ can be better probed through $\mathrm{t}$-channel $\mathrm{W}$-W and/or Z-Z fusion at high energy $e^{+} e^{-}$collisions $e^{+} e^{-} \rightarrow t \bar{c} \nu_{2} \bar{\nu}_{e}$ and $e^{+} e^{-} \rightarrow t \bar{c} e^{+} e^{-}$ $[54,55]$. An interesting feature of these reactions is that, being t-channel fusion processes, their cross sections grow with energy unlike s-channel reactions $e^{+} e^{-} \rightarrow t \bar{c}$ which are suppressed at high energies. The cross sections of $e^{+} e^{-} \rightarrow t \bar{c} \nu_{e} \bar{\nu}_{e}$ and $e^{+} e^{-} \rightarrow t \bar{c} e^{+} e^{-}$are found to be one or two orders of magnitude bigger than the cross sections of $e^{+} e^{-} \rightarrow t \bar{c}$ $[54,55]$.

In the present study, we limit ourself to $e^{+} e^{-}$colliders, its $\gamma \gamma$ option and muon colliders. The cross sections for $e^{+} e^{-} \rightarrow \bar{t} c, \gamma \gamma \rightarrow \bar{t} c$ and/or $\mu^{+} \mu^{-} \rightarrow \bar{t} c$ in 2HDM, if sizeable, can give information on the top FCNC couplings $t \rightarrow c \gamma$ and $t \rightarrow c Z$ as well as Higgs FCNC $\Phi \rightarrow \bar{t} c$. Unlike the process $e^{+} e^{-} \rightarrow \bar{t} c$, which is s-channel suppressed at high energy, the process $\gamma \gamma \rightarrow \bar{t} c$ has a t and u-channel contributions, its cross section may grow with the center of mass energy.

At $e^{+} e^{-}$collisions, the generic contributions to $e^{+} e^{-} \rightarrow \bar{t} c$ are drawn in Fig. (9). We have three sets of diagrams: $\gamma \bar{t} c$ vertex contributions through $e^{+} e^{-} \rightarrow \gamma^{*} \rightarrow \bar{t} c, Z \bar{t} c$ vertex contributions through $e^{+} e^{-} \rightarrow Z^{*} \rightarrow \bar{t} c$ and box contributions. The topological contributions to $e^{+} e^{-} \rightarrow \bar{t} c$ are depicted in Fig .(9).

In $\gamma \gamma$ collisions, we have more topological diagrams that contribute to $\gamma \gamma \rightarrow \bar{t} c . \gamma \bar{t} c$ vertex enters game through diagrams $d_{2}, d_{3}, d_{7}$ and $d_{8}$ of Fig. (10) and their crossed one. The quartic coupling of 2 photons to a pair of $H^{+} H^{-}$or $W^{+} W^{-}$gives the topological diagram $d_{1}$. Box (resp self-energies) topologies are depicted in diagrams $d_{4}$ and $d_{5}$ (resp $d_{6}$ ). We have checked both analytically and numerically that the result is Ultra-Violet finite and renormalization scale independent. In the case of $\gamma \gamma \rightarrow \bar{t} c$, gauge invariance has been checked numerically.

Due to GIM mechanism, in the SM, the cross sections of $e^{+} e^{-} \rightarrow \bar{t} c$ and $\gamma \gamma \rightarrow \bar{t} c$ are very suppressed see Fig. (11) (left and right). It is clear from Fig. (11) that, in the SM, the $\gamma \gamma \rightarrow \bar{t} c$ cross section is about one order of magnitude bigger than $e^{+} e^{-} \rightarrow \bar{t} c$ cross 

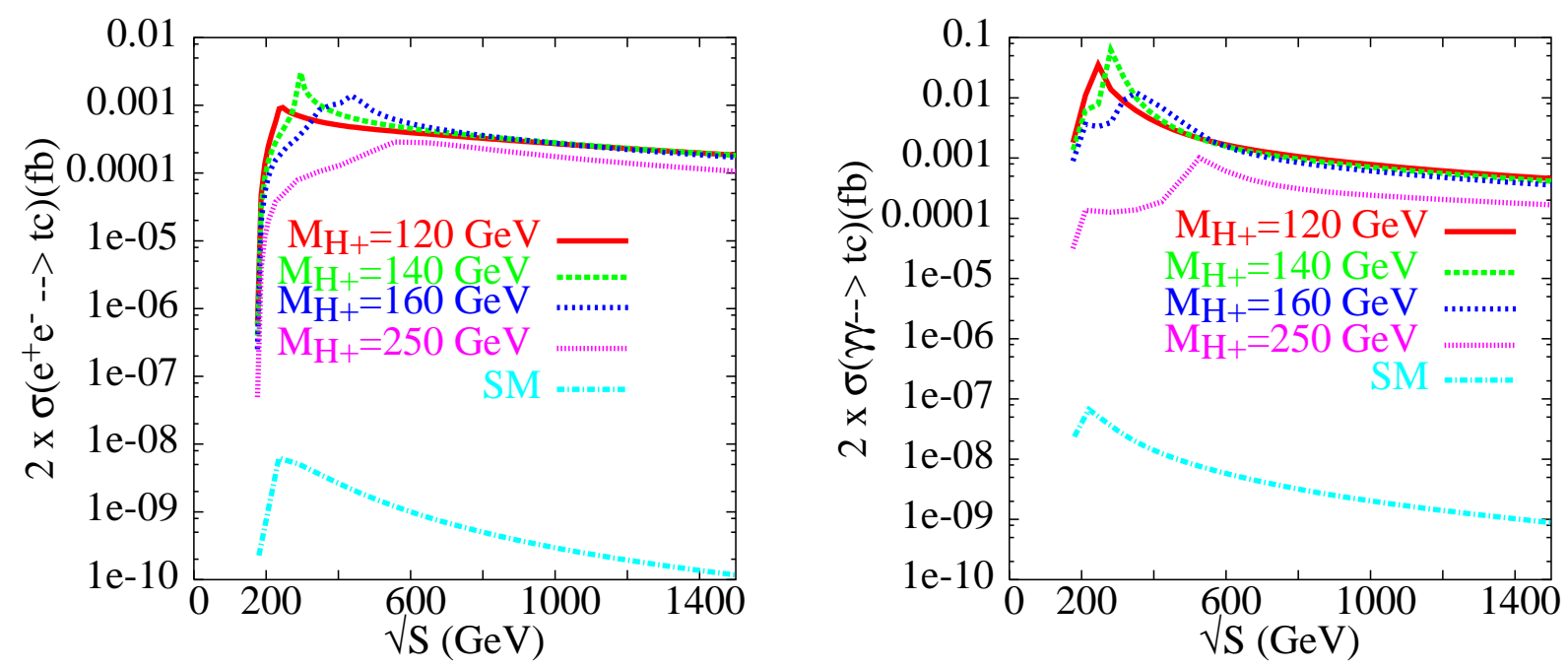

Figure 11: Cross sections for associated top-charm production at $e^{+} e^{-}$colliders (left) and its $\gamma \gamma$ option (right) in 2HDM-II with $\tan \beta=65$ and several choices of charged Higgs mass

sections. Our results for the SM agree with [51]

We illustrate our numerical results in 2HDM-II for large $\tan \beta=65$ and several values of charged Higgs. The reason for this choice is the fact that the one loop effective coupling $t \rightarrow c \gamma$ and $t \rightarrow c Z$ are larger for large $\tan \beta$ (see Fig. (2)). We consider only unpolarized cross sections. It is well known that the cross sections for polarized initial states differ from the unpolarized cross sections only by the normalization factor.

In both cases, for $e^{+} e^{-}$and $\gamma \gamma$ collisions, the cross sections in the 2HDM are enhanced by about four orders of magnitude with respect to their SM values. Above $\sqrt{s}=200 \mathrm{GeV}$, the cross sections become sizeable and develop a peak at $\sqrt{s}=2 M_{H \pm}$, which is due to the threshold effect of charged Higgs pair production. The cross sections can reach a value of $0.003 \mathrm{fb}(\operatorname{resp} 0.1 \mathrm{fb})$ for $e^{+} e^{-} \rightarrow \bar{t} c(\operatorname{resp} \gamma \gamma \rightarrow \bar{t} c)$ near threshold. At $\gamma \gamma$ options, cross sections of about $0.01 \rightarrow 0.1 \mathrm{fb}$ would give $4 \rightarrow 80$ events for the assumed luminosities of $400 \rightarrow 800 \mathrm{fb}^{-1}$. Above the threshold region $\sqrt{s} \gtrsim 800 \mathrm{GeV}$, the cross section decreases with increasing the energy and reaches a value of $\approx 10^{-4} \mathrm{fb}$ at $\sqrt{s} \gtrsim 1.5 \mathrm{TeV}$.

In the $2 \mathrm{HDM}-\mathrm{I}$, the cross sections are below $\approx 10^{-3} \mathrm{fb}\left(\operatorname{resp} 10^{-5} \mathrm{fb}\right)$ for $\gamma \gamma\left(\operatorname{resp} e^{+} e^{-}\right)$ collisions.

In recent years an increasing amount of work has been dedicated to the physics possibilities of $\mu^{+} \mu^{-}$colliders [3]. Such colliders offer novel ways of producing Higgs bosons, such as an $s$-channel resonance in the case of neutral scalars. The production process $\mu^{+} \mu^{-} \rightarrow \bar{t} c$ proceed through the topological diagrams drawn in Fig. 9. As for $e^{+} e^{-} \rightarrow \bar{t} c$ we have the gauge boson s-channel $\mu^{+} \mu^{-} \rightarrow \gamma, Z^{*} \rightarrow \bar{t} c$ contributions as well as the Higgs boson s-channel exchange $\mu^{+} \mu^{-} \rightarrow h^{*}, H^{*}, A^{*} \rightarrow \bar{t} c$. The three couplings $h^{0} \mu^{+} \mu^{-}, H^{0} \mu^{+} \mu^{-}$ and $A^{0} \mu^{+} \mu^{-}$behave like $m_{\mu} \tan \beta$ at large $\tan \beta$ and this might be a source of enhancement in the large $\tan \beta$ limit. Such enhancement has been already observed for $\mu^{+} \mu^{-} \rightarrow W^{ \pm} H^{\mp}$ 

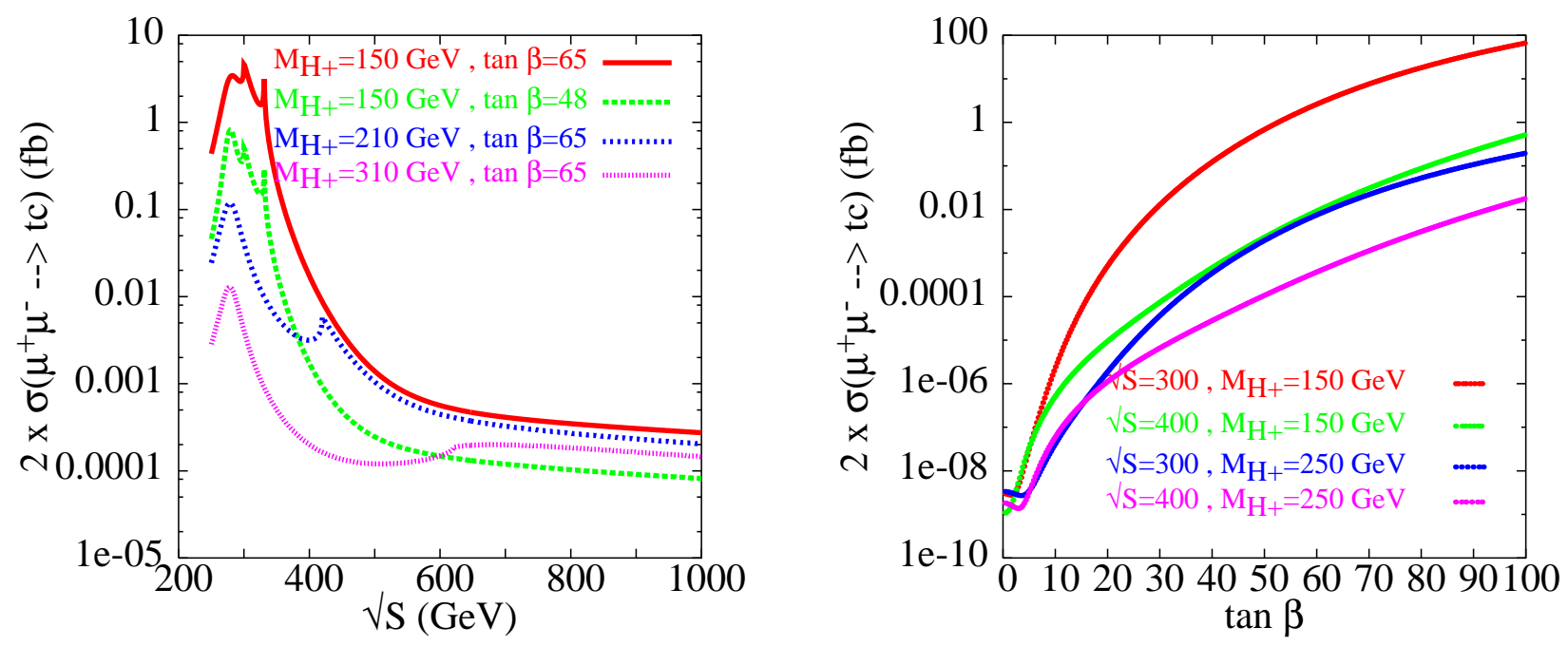

Figure 12: $\mu^{+} \mu^{-} \rightarrow t \bar{c}+\bar{t} c$ cross section as function of $\sqrt{s}$ (left) and $\tan \beta$ (right) for $M_{h}=100, M_{H}=278, M_{A}=170 \mathrm{GeV}, \sin \alpha=-0.84$ and $\lambda_{5}=M_{A}^{2} / v^{2}=0.95$.

and $\mu^{+} \mu^{-} \rightarrow Z A^{0}[56]$.

In the SM, it is expected that the cross section $\mu^{+} \mu^{-} \rightarrow \bar{t} c$ is very suppressed as in the case for $e^{+} e^{-} \rightarrow \bar{t} c$. In the case of $\mu^{+} \mu^{-} \rightarrow \bar{t} c$, the only difference with respect to $e^{+} e^{-} \rightarrow \bar{t} c$ is the contribution of Higgs boson s-channel $\mu^{+} \mu^{-} \rightarrow H^{*} \rightarrow \bar{t} c$. However, in SM the couplings $H^{0} \mu^{+} \mu^{-}$as well as $H^{0} \bar{t} c$ does not have any enhancement factor like $\tan \beta$. Therefore, the cross section for $\mu^{+} \mu^{-} \rightarrow \bar{t} c$ will be similar in magnitude to the $e^{+} e^{-} \rightarrow \bar{t} c$ cross section.

We show in Fig. (12) cross sections for $\mu^{+} \mu^{-} \rightarrow \bar{t} c$ as a function of center of mass energy (left) and as a function of $\tan \beta$ (right). As expected, the cross sections are enhanced for large $\tan \beta$ where both $\left\{h^{0}, H^{0}, A^{0}\right\} \mu^{+} \mu^{-}$and $\left\{h^{0}, H^{0}, A^{0}\right\} \bar{t} c$ couplings are enhanced. As can be seen from right plot of Fig. 12, the cross section can reach a few $\mathrm{fb}$ near threshold region for $\tan \beta=65$ and light charged Higgs. One can see also several kinks, the first one on the left is located around $\sqrt{s} \approx M_{H}=278 \mathrm{GeV}$ and is due to s-channel resonance of the heavy CP-even Higgs $H^{0}$. The second kink, located at several points where $\sqrt{s} \approx 2 M_{H \pm}$, is due to the threshold production of pair of charged Higgs. For Fig. 12, instead of using unitarity constraints we have used the perturbativity constraints on the $\lambda_{i}: \lambda_{i} \lesssim 8 \pi$. That has been done in order to allow a larger reach for $\tan \beta$. For instance, for charged Higgs of $150 \mathrm{GeV}$, unitarity constraints are satisfied only for $\tan \beta \lesssim 48$ while perturbativity constraints on the $\lambda_{i}$ are satisfied even for $\tan \beta \approx 100$. As it can be seen from those plots, the cross section can be larger than $0.01 \mathrm{fb}$ only for low center of mass energy $\sqrt{s} \lesssim 500$ $\mathrm{GeV}$ and rather light charged Higgs. For high center of mass energy $\sqrt{s} \gtrsim 500 \mathrm{GeV}$, the cross sections of the s-channel process $\mu^{+} \mu^{-} \rightarrow \bar{t} c$ are reduced.

We note that in 2HDM-I, $\mu^{+} \mu^{-} \rightarrow \bar{t} c$ would not register any observable signal. This is due to the fact that some fermionic couplings are proportional to $\cot ^{2} \beta$, and so unacceptably small values of $\tan \beta$ would be required in order to allow observable cross-sections. 


\section{Conclusions}

In the framework of $2 \mathrm{HDM}$ with natural flavor conservation, we have studied various top and Higgs flavor changing neutral couplings: $t \rightarrow c \gamma, c Z, c g, c h^{0}$ and $\Phi \rightarrow \bar{t} c$. The study has been carried out taking into account the experimental constraint on the $\rho$ parameter, unitarity constraints, and vacuum stability conditions on all scalar quartic couplings $\lambda_{i}$. Numerical results for the branching ratios have been presented. We emphasize the effect coming from bottom Yukawa couplings and pure trilinear scalar couplings such as $h^{0} H^{+} H^{-}$ and $H^{0} H^{+} H^{-}$.

We have shown that, in 2HDM-II, the branching ratios of top FCNC decays $t \rightarrow c \gamma, c Z, c g, c h^{0}$ as well as Higgs FCNC $\left\{h^{0}, H^{0}\right\} \rightarrow \bar{t} c$ can be orders of magnitude larger than their SM values. Such enhancement requires large $\tan \beta$, rather light charged Higgs and not too heavy CP-even Higgs masses. We have shown that $t \rightarrow c h^{0}$ as well as $\left\{h^{0}, H^{0}\right\} \rightarrow \bar{t} c$ can reach observable rates at LHC with Branching ratios of the order $10^{-5}$ while all the unitarity and vacuum stability constraints are fulfilled. With Branching ratio of this size, the production and subsequent decay of the neutral CP-even Higgs mediated by FCNC can lead to a few hundred events at LHC with the High luminosity option [15].

We have also studied the top-charm associated production at $e^{+} e^{-}$colliders, its $\gamma \gamma$ option and also at muon colliders. It has been shown that, in 2HDM-II, the cross section of $\gamma \gamma \rightarrow \bar{t} c$ can be of the order $0.01 \rightarrow 0.1 \mathrm{fb}$ near the threshold region while the corresponding cross sections at $e^{+} e^{-}$remain well below $10^{-2} \mathrm{fb}$. At muon colliders, the situation is slightly better due to Higgs boson $h^{0}, H^{0}$ and $A^{0}$ s-channel contribution. We showed that $\mu^{+} \mu^{-} \rightarrow \bar{t} c$ production offers an attractive way of searching for FCNC at such colliders. The cross section grows with increasing $\tan \beta$ with values of few $\mathrm{fb}$ being attainable for $\tan \beta \geq 48$.

Acknowledgments This work is supported by the Physics Division of National Center for Theoretical Sciences under a grant from the National Science Council of Taiwan. We are grateful to A. Akeroyd for useful discussions and for reading the paper. We thank K. Cheung and O. Kong for comments about $(g-2)_{\mu}$. We would like to thank J. Guasch and S. Béjar for exchange of informations about Ref. [15].

\section{References}

[1] M. Beneke, I. Efthymipopulos, M. L. Mangano, J. Womersley (conveners) et al., report in the Workshop on Standard Model Physics (and more) at the LHC, Geneva, hep$\mathrm{ph} / 0003033$

[2] J. A. Aguilar-Saavedra et al. [ECFA/DESY LC Physics Working Group Collaboration], arXiv:hep-ph/0106315. T. Abe et al. [American Linear Collider Working Group], in Proc. of the APS/DPF/DPB Summer Study on the Future of Particle Physics 
(Snowmass 2001) ed. N. Graf, arXiv:hep-ex/0106058. K. Abe et al. [ACFA Linear Collider Working Group], arXiv:hep-ph/0109166.

[3] V. D. Barger, M. S. Berger, J. F. Gunion and T. Han, Phys. Rept. 286, 1 (1997) [arXiv:hep-ph/9602415].

[4] G. Eilam, J. L. Hewett and A. Soni, Phys. Rev. D 44 (1991) 1473 [Erratum-ibid. D 59 (1999) 039901].

[5] B. Mele, S. Petrarca and A. Soddu, Phys. Lett. B435, 401 (1998)

[6] F. Abe et al. [CDF Collaboration], Phys. Rev. Lett. 80, 2525 (1998) M. Paulini [CDF and D0 Collaborations], arXiv:hep-ex/9701019. D. W. Gerdes, arXiv:hep-ex/9706001.

[7] V. F. Obraztsov, Prepared for 30th International Conference on High-Energy Physics (ICHEP 2000), Osaka, Japan, 27 Jul - 2 Aug 2000;

R. Barate et al. [ALEPH Collaboration], CERN-EP-2000-102

[8] J. A. Aguilar-Saavedra, Acta Phys. Polon. B 35, 2695 (2004) [arXiv:hep-ph/0409342].

[9] J. A. Aguilar-Saavedra and B. M. Nobre, Phys. Lett. B 553, 251 (2003) [arXiv:hep$\mathrm{ph} / 0210360]$.

[10] F. del Aguila, J. A. Aguilar-Saavedra and R. Miquel, Phys. Rev. Lett. 82, 1628 (1999) F. del Aguila and M. J. Bowick, Nucl. Phys. B224, 107 (1983); K. Higuchi and K. Yamamoto, Phys. Rev. D 62, 073005 (2000)

[11] D. Atwood, L. Reina and A. Soni, Phys. Rev. D55, 3156 (1997)

[12] W. S. Hou, Phys. Lett. B 296, 179 (1992).

[13] M. E. Luke and M. J. Savage, Phys. Lett. B 307, 387 (1993). R. A. Diaz, R. Martinez and J. Alexis Rodriguez, arXiv:hep-ph/0103307.

[14] S. Bejar, J. Guasch and J. Sola, Nucl. Phys. B 600, 21 (2001) [arXiv:hep-ph/0011091].

[15] S. Bejar, J. Guasch and J. Sola, Nucl. Phys. B 675, 270 (2003) [arXiv:hep$\mathrm{ph} / 0307144]$.

[16] A. Arhrib, Phys. Lett. B 612, 263 (2005) [arXiv:hep-ph/0409218].

[17] S. Kanemura, K. Matsuda, T. Ota, T. Shindou, E. Takasugi and K. Tsumura, Phys. Lett. B 599, 83 (2004). S. Kanemura, T. Ota and K. Tsumura, arXiv:hep-ph/0505191. S. Kanemura, T. Ota and K. Tsumura, arXiv:hep-ph/0509005.

[18] J. M. Yang, B. Young and X. Zhang, Phys. Rev. D58, 055001 (1998) G. Eilam, A. Gemintern, T. Han, J. M. Yang and X. Zhang, Phys. Lett. B 510, 227 (2001) [arXiv:hep-ph/0102037]. 
[19] C. S. Li, R. J. Oakes and J. M. Yang, Phys. Rev. D 49, 293 (1994) [Erratum-ibid. D 56, 3156 (1997)]. G. Couture, C. Hamzaoui and H. Konig, Phys. Rev. D 52, 1713 (1995) [arXiv:hep-ph/9410230]. J. L. Lopez, D. V. Nanopoulos and R. Rangarajan, Phys. Rev. D 56, 3100 (1997) [arXiv:hep-ph/9702350]. G. M. de Divitiis, R. Petronzio and L. Silvestrini, Nucl. Phys. B 504, 45 (1997). J. M. Yang and C. S. Li, Phys. Rev. D 49, 3412 (1994) [Erratum-ibid. D 51, 3974 (1995)]. D. Delepine and S. Khalil, Phys. Lett. B 599, 62 (2004). J. J. Liu, C. S. Li, L. L. Yang and L. G. Jin, Phys. Lett. B 599, $92(2004)$.

[20] J. Guasch and J. Sola, Nucl. Phys. B 562, 3 (1999) [arXiv:hep-ph/9906268].

[21] A. M. Curiel, M. J. Herrero, W. Hollik, F. Merz and S. Penaranda, Phys. Rev. D 69, 075009 (2004) [arXiv:hep-ph/0312135]. A. M. Curiel, M. J. Herrero and D. Temes, Phys. Rev. D 67, 075008 (2003) [arXiv:hep-ph/0210335]. D. A. Demir, Phys. Lett. B 571, 193 (2003) [arXiv:hep-ph/0303249].

[22] J. A. Aguilar-Saavedra and G. C. Branco, Phys. Lett. B 495, 347 (2000) [arXiv:hepph/0004190]. J. A. Aguilar-Saavedra, Phys. Lett. B 502, 115 (2001) [arXiv:hep$\mathrm{ph} / 0012305]$.

[23] S. Weinberg, Phys. Rev. Lett. 37, 657 (1976).

[24] for recent discussion on CP violation in 2HDM see: J. F. Gunion and H. E. Haber, arXiv:hep-ph/0506227.

[25] H. J. He, C. T. Hill and T. M. Tait, Phys. Rev. D 65, 055006 (2002) [arXiv:hep$\mathrm{ph} / 0108041]$.

[26] For a review see e.g. J. F. Gunion, H. E. Haber, G. L. Kane and S. Dawson, SCIPP89/13 and ERRATA, hep-ph/9302272;

[27] J. F. Gunion and H. E. Haber, Phys. Rev. D 67, 075019 (2003) [arXiv:hepph/0207010]. L. Brucher and R. Santos, Eur. Phys. J. C 12, 87 (2000) [arXiv:hep$\mathrm{ph} / 9907434]$.

[28] A. G. Akeroyd, A. Arhrib and E. Naimi, Eur. Phys. J. C 20, 51 (2001) [arXiv:hepph/0002288]. A. Arhrib and G. Moultaka, Nucl. Phys. B 558, 3 (1999) [arXiv:hep$\mathrm{ph} / 9808317]$.

[29] M. Sher, Phys. Rept. 179, 273 (1989). S. Kanemura, T. Kasai and Y. Okada, Phys. Lett. B 471, 182 (1999) [arXiv:hep-ph/9903289].

[30] A. Barroso, P. M. Ferreira and R. Santos, arXiv:hep-ph/0507329. P. M. Ferreira, R. Santos and A. Barroso, Phys. Lett. B 603, 219 (2004) [arXiv:hep-ph/0406231].

[31] B. W. Lee, C. Quigg and H. B. Thacker, Phys. Rev. Lett. 38, 883 (1977). 
[32] S. Kanemura, T. Kubota, E. Takasugi, Phys. Lett. B313, 155 (1993);

[33] A. G. Akeroyd, A. Arhrib, E. M. Naimi, Phys. Lett. B490, 119 (2000); A. Arhrib, hep-ph/0012353.

[34] K. Hagiwara et al. [Particle Data Group Collaboration], Phys. Rev. D 66 (2002) 010001.

[35] P. Q. Hung, R. McCoy and D. Singleton, Phys. Rev. D 50, 2082 (1994).

[36] V. D. Barger, J. L. Hewett and R. J. Phillips, Phys. Rev. D 41, 3421 (1990); Y. Grossman, Nucl. Phys. B 426, 355 (1994) [arXiv:hep-ph/9401311].

[37] P. Gambino and M. Misiak, Nucl. Phys. B611, 338 (2001); F. M. Borzumati and C. Greub, Phys. Rev. D58, 074004 (1998); ibid Phys. Rev. D59, 057501 (1999);

[38] M. Neubert, Eur. Phys. J. C 40, 165 (2005) [arXiv:hep-ph/0408179].

[39] G. W. Bennett et al. [Muon g-2 Collaboration], Phys. Rev. Lett. 92, 161802 (2004) [arXiv:hep-ex/0401008]. H. Deng [Muon g-2 Collaboration], eConf C0406271, TUET03 (2004) [arXiv:hep-ex/0408148].

[40] A. Dedes and H. E. Haber, JHEP 0105, 006 (2001) [arXiv:hep-ph/0102297].

[41] M. Krawczyk and J. Zochowski, Phys. Rev. D 55, 6968 (1997) [arXiv:hep-ph/9608321].

[42] D. Chang, W. F. Chang, C. H. Chou and W. Y. Keung, Phys. Rev. D 63, 091301 (2001) [arXiv:hep-ph/0009292].

[43] K. m. Cheung, C. H. Chou and O. C. W. Kong, Phys. Rev. D 64, 111301 (2001) [arXiv:hep-ph/0103183]; K. Cheung and O. C. W. Kong, Phys. Rev. D 68, 053003 (2003) [arXiv:hep-ph/0302111].

[44] G. Abbiendi et al. [OPAL Collaboration], Eur. Phys. J. C 18, 425 (2001) [arXiv:hepex/0007040].

[45] T. Hahn, Comput. Phys. Commun. 140, 418 (2001); T. Hahn, C. Schappacher, Comput. Phys. Commun. 143, 54 (2002); T. Hahn, M. Perez-Victoria, Comput. Phys. Commun. 118, 153 (1999); J. Küblbeck, M. Böhm, A. Denner, Comput. Phys. Commun. 60, 165 (1990);

[46] G. J. van Oldenborgh, Comput. Phys. Commun. 66, 1 (1991); T. Hahn, Acta Phys. Polon. B 30, 3469 (1999)

[47] S. Bejar, F. Dilme, J. Guasch and J. Sola, JHEP 0408, 018 (2004) [arXiv:hep$\mathrm{ph} / 0402188]$.

[48] A. Djouadi, arXiv:hep-ph/0503173. 
[49] D. Atwood, L. Reina and A. Soni, Phys. Rev. D 53, 1199 (1996). H. J. He, S. Kanemura and C. P. Yuan, Phys. Rev. Lett. 89, 101803 (2002). H. J. He and C. P. Yuan, Phys. Rev. Lett. 83, 28 (1999) [arXiv:hep-ph/9810367].

[50] S. Bejar, J. Guasch and J. Sola, arXiv:hep-ph/0508043.

[51] C. S. Huang, X. H. Wu and S. H. Zhu, Phys. Lett. B 452, 143 (1999) [arXiv:hep$\mathrm{ph} / 9901369]$.

[52] C. S. Li, X. m. Zhang and S. H. Zhu, Phys. Rev. D 60, 077702 (1999) [arXiv:hep$\mathrm{ph} / 9904273]$.

[53] D. Atwood, L. Reina and A. Soni, Phys. Rev. Lett. 75, 3800 (1995) [arXiv:hep$\mathrm{ph} / 9507416]$.

[54] S. Bar-Shalom, G. Eilam, A. Soni and J. Wudka, Phys. Rev. D 57, 2957 (1998) [arXiv:hep-ph/9708358].

[55] W. S. Hou, G. L. Lin and C. Y. Ma, Phys. Rev. D 56, 7434 (1997) [arXiv:hep$\mathrm{ph} / 9708228]$.

[56] A. G. Akeroyd, A. Arhrib and C. Dove, Phys. Rev. D 61, 071702 (2000) [arXiv:hepph/9910287]. A. G. Akeroyd and S. Baek, Phys. Lett. B 500, 142 (2001) [arXiv:hep$\mathrm{ph} / 0008286]$. 